\title{
Neu3 Sialidase-Mediated Ganglioside Conversion Is Necessary for Axon Regeneration and Is Blocked in CNS Axons
}

\author{
Sunil Kappagantula, ${ }^{1}$ Melissa R. Andrews, ${ }^{1,2}$ Menghon Cheah, ${ }^{1}$ José Abad-Rodriguez, ${ }^{3}$ Carlos G. Dotti, ${ }^{4}$ \\ and James W. Fawcett ${ }^{1}$ \\ ${ }^{1}$ John van Geest Centre for Brain Repair, University of Cambridge, Cambridge, CB2 0PY, United Kingdom, ${ }^{2}$ School of Medicine, University of St. Andrews, \\ St. Andrews, KY16 9TF, United Kingdom, ${ }^{3}$ Membrane Biology and Axonal Repair Laboratory Hospital Nacional de Parapléjicos, E-45071 Toledo, Spain, \\ and ${ }^{4}$ Centro de Biología Molecular Severo Ochoa, 28049, Madrid, Spain
}

PNS axons have a high intrinsic regenerative ability, whereas most CNS axons show little regenerative response. We show that activation of Neu3 sialidase, also known as Neuraminidase-3, causing conversion of GD1a and GT1b to GM1 ganglioside, is an essential step in regeneration occurring in PNS (sensory) but not CNS (retinal) axons in adult rat. In PNS axons, axotomy activates Neu3 sialidase, increasing the ratio of GM1/GD1a and GM1/GT1b gangliosides immediately after injury in vitro and in vivo. No change in the GM1/GD1a ratio after axotomy was observed in retinal axons (in vitro and in vivo), despite the presence of Neu3 sialidase. Externally applied sialidase converted GD1a ganglioside to GM1 and rescued axon regeneration in CNS axons and in PNS axons after Neu3 sialidase blockade. Neu3 sialidase activation in DRGs is initiated by an influx of extracellular calcium, activating P38MAPK and then Neu3 sialidase. Ganglioside conversion by Neu3 sialidase further activates the ERK pathway. In CNS axons, P38MAPK and Neu3 sialidase were not activated by axotomy.

Key words: axon regeneration; axotomy; gangliosides; Neu3 sialidase

\section{Introduction}

Mammalian CNS axons fail to regenerate after damage, whereas PNS axons can regenerate vigorously, many reconnecting with their targets. Mature CNS axons have been shown to be intrinsically poor at regenerating even in permissive environments (Sun and He, 2010; Moore and Goldberg, 2011; Wang and Jin, 2011; Chew et al., 2012; Cho and Cavalli, 2012). Successful axon regeneration begins with growth cone formation involving coordinated proteolytic, cytoskeletal, and cell surface regulatory events (Bradke and Dotti, 1999; Long and Lemmon, 2000; Erez et al., 2007; Witte et al., 2008; Afshari et al., 2009; Bradke et al., 2012) as well as activation of local protein translation as in PNS axons (Zheng et al., 2001; Verma et al., 2005). Integrins and other signaling molecules involved with control of regeneration can asso-

Received Oct. 3, 2013; revised Dec. 9, 2013; accepted Dec. 23, 2013.

Author contributions:S.K., M.R.A., J.A.-R., C.G.D., and J.W.F. designed research; S.K., M.R.A., and M.C. performed research; S.K., M.R.A., J.A.-R., C.G.D., and J.W.F. analyzed data; S.K., M.R.A., M.C., J.A.-R., C.G.D., and J.W.F. wrote the paper.

This work was supported by the Medical Research Council, the Christopher and Dana Reeve Foundation, the John and Lucille van Geest Foundation, the Henry Smith Charity, the Commonwealth and Overseas scholarships, and the Hinduja Cambridge Trust. We thank Prof. Ronald L. Schnaar (Johns Hopkins University) for generously providing anti-GD1a ganglioside antibody and Mr. David Story and Mr. Marc Smith (Cambridge University) for technical assistance.

J.W.F. is a paid advisor to Acorda Therapeutics, Novartis, and Covidien. The remaining authors declare no competing financial interests.

Correspondence should be addressed to Dr. James W. Fawcett, John van Geest Centre for Brain Repair, University of Cambridge, Forvie Site, Robinson Way, Cambridge, CB2 OPY, United Kingdom. E-mail: jf108@cam.ac.uk.

DOI:10.1523/JNEUROSCI.4432-13.2014

Copyright $\odot 2014$ the authors $\quad 0270-6474 / 14 / 342477-16 \$ 15.00 / 0$ ciate with lipid microdomains, leading to modulation of many intracellular signaling events (Guirland et al., 2004; Kamiguchi, 2006; Guirland and Zheng, 2007; Grider et al., 2009; Ohkawa et al., 2010; Stuermer, 2010; Gao et al., 2011; Norambuena and Schwartz, 2011).

Gangliosides, complex glycosphingolipids on the plasma membrane containing one or more sialic acid residues, are key components of microdomains. The adult mammalian nervous system has four dominant types of gangliosides: GM1, GD1a, GD1b, and GT1b. Whereas embryos can develop normally without complex gangliosides, postnatally they develop demyelination, neurodegeneration, and impaired peripheral nerve regeneration (Sheikh et al., 1999; Okada et al., 2002; Kittaka et al., 2008). GM1 ganglioside has been shown to promote axon growth (Facci et al., 1984; Skaper et al., 1985) and regeneration (Yang et al., 2006; Mountney et al., 2010), but a clinical trial of GM1 treatment in spinal cord injury was not successful (Geisler et al., 2001). GD1a ganglioside, on the other hand, has been shown to be a receptor for inhibition of axon growth by MAG (Vyas et al., 2002; Zhang et al., 2011), and GT1b forms a complex with the Nogo receptor to inhibit axon growth (Williams et al., 2008). Neu3 sialidase, an enzyme that hydrolyzes polysialic acid-containing gangliosides to GM1 ganglioside (Geisler et al., 2001; Vyas et al., 2002), acts on outer sialic acid groups and has been shown to effectively increase GM1 levels (Oehler et al., 2002; Monti and Miyagi, 2012). Additionally, Neu3 has been shown to be necessary for neuronal differenti- 
ation (Da Silva et al., 2005), for maintenance of polarity after axotomy (Rodriguez et al., 2001), and for regulating invasive and migratory activity (Yamaguchi et al., 2006; Miyata et al., 2011; Tringali et al., 2012).

In this paper, we examine Neu3 sialidase action on axonal gangliosides and resultant axon regeneration, and we assess whether these mechanisms help to explain the different regeneration capacities of CNS and PNS axons. We find that after PNS axotomy, but not CNS, Neu3 sialidase converts GD1a to GM1. Pharmacological blocking of Neu3 sialidase, downregulation of Neu3 with siRNA, or depleting gangliosides all inhibit PNS regeneration. Conversely, exogenously applied sialidase increases GM1 ganglioside and promotes regeneration in CNS axons, suggesting that conversion of polysialyated gangliosides is necessary for axon regeneration.

\section{Materials and Methods Chemicals and reagents}

All chemicals, antibodies, and reagents were purchased from SigmaAldrich, unless otherwise specified.

\section{DRG cultures}

Adult (3- to 4-month-old) male Sprague Dawley rats were euthanized and DRGs removed.

\section{Explants}

DRGs were trimmed of meningeal coverings, divided into segments, and plated onto coated chamber slides (Nunc) $[10 \mu \mathrm{g} / \mathrm{ml}$ poly-D-lysine (PDL) and $1 \mu \mathrm{g} / \mathrm{ml} \mathrm{laminin].} \mathrm{Explants} \mathrm{were} \mathrm{maintained} \mathrm{at} 37^{\circ} \mathrm{C}$ and $7 \%$ $\mathrm{CO}_{2}$ in DMEM (Invitrogen) with $0.11 \mathrm{~g} / \mathrm{L}$ sodium pyruvate with pyrodixine, $4.5 \mathrm{~g} / \mathrm{L}$ glucose, $20 \mathrm{ng} / \mathrm{ml} \mathrm{NGF}, 1 \%$ insulin-transferrin-selenium, $1 \%$ penicillin-streptomycin-fungizone.

\section{Dissociated DRGs}

DRGs were dissociated with $0.2 \%$ collagenase followed by $0.1 \%$ trypsin and triturated with Pasteur pipettes of decreasing tip diameters. The suspension was centrifuged through a density gradient (15\% BSA). Cells were cultured in DMEM supplemented with 10\% FBS (Invitrogen), 1\% penicillin-streptomycin-fungizone, $0.1 \%$ mitomycin- $C$, and $10 \mathrm{ng} / \mathrm{ml}$ NGF and plated on PDL $(20 \mu \mathrm{g} / \mathrm{ml})+$ laminin $(10 \mu \mathrm{g} / \mathrm{ml})$.

\section{Retina explant cultures}

Unilateral conditioning optic nerve crush was performed on adult male Sprague Dawley rats, $7 \mathrm{~d}$ before retinal dissection. Retinas were separated from underlying pigment epithelium and sclera in HBSS before mounting flat onto Petri dishes and cutting into $400-\mu \mathrm{m}$-thick sections using a Mcllwain tissue chopper (Vibratome). Retinal sections were plated onto plastic chamber slides coated with $300 \mu \mathrm{g} / \mathrm{ml}$ PDL and $10 \mu \mathrm{g} / \mathrm{ml} \mathrm{laminin,}$ with the ganglion cell layer facing the substratum. Cultures were maintained in DMEM and Neurobasal-A (Invitrogen) in a 1:1 ratio, supplemented with 50 IU erythropoietin-A, $760 \mu \mathrm{g} / \mathrm{ml}$ BSA, N2 supplement (Invitrogen ), $100 \mu \mathrm{g} / \mathrm{ml}$ sodium pyruvate, $4 \mu \mathrm{g} / \mathrm{ml}$ of T3-T4, $2 \mathrm{~mm}$ glutamine (Invitrogen), $1.1 \mathrm{mg} / \mathrm{ml}$ glucose, $100 \mu \mathrm{g} / \mathrm{ml}$ gentamycin (Invitrogen), and $50 \mathrm{ng} / \mathrm{ml}$ ciliary neurotrophic factor.

\section{Axon regeneration assay}

Axons were grown for 48-72 h (DRGs) or 5-7 d (retina explants) and then axotomized with a pulled glass electrode leaving the site of axotomy clearly demarcated on the plastic chamber slide. Axons were photographed immediately, $15 \mathrm{~min}$, and $1 \mathrm{~h}$ after injury and assessed for regeneration. Images were captured on a Nikon phase-contrast ELWD 0.3 microscope equipped with a Nikon DXM 1200 digital camera using a $10 \times(10 \times / 0.25)$ or $20 \times(20 \times / 0.4)$ objective lens. For analysis, only cut axons that had retracted and begun to grow back were regarded as regenerating axons.

\section{Pharmacological treatments}

Inhibition of ganglioside synthesis. Axons were treated with either fumonisin B1 $(3 \mu \mathrm{M})$ or $( \pm)$-threo-1-phenyl-2-decanoylamino-3morpholino-1-propanol hydrochloride (PDMP) $(20 \mu \mathrm{M}) 18 \mathrm{~h}$ before axotomy.

Modulation of Neu3 sialidase activity. Axons were treated with 2,3, dehydro-2,deoxy- $N$-acetylneuraminic acid (NeuAc2en; $800 \mu \mathrm{M}$ ) for inhibition of Neu3 sialidase activity and/or with exogenous Clostridium sialidase (0.4 IU; Roche), immediately before axotomy. NeuAc2en was removed before adding Clostridium sialidase as NeuAc2en inhibited Clostridium sialidase activity.

Modulation of signaling pathways. To analyze the regulation of Neu3 sialidase activity after adult DRG axotomy, one of the following was added to the media 30 min before axotomy and left in the media for a further 60 min: U0126 (MEK-ERK inhibitor, $20 \mu \mathrm{M}$, Promega), rapamycin (mTOR inhibitor, $10 \mathrm{~nm}$, Calbiochem), and SB203580 (P38MAPK inhibitor, $5 \mu \mathrm{M}$, Calbiochem). Anisomycin (10 $\mu \mathrm{M}$, Alomone Labs) was used to activate P38MAPK.

To examine the effect of absence of $\mathrm{eCa}^{2+}$ on Neu3 sialidase activity and axonal regeneration, cultures were incubated with normal growth media devoid of $\mathrm{CaCl}_{2}, 30$ min before axotomy.

\section{siRNA transfection}

Dissociated DRGs were transfected with siRNA $48 \mathrm{~h}$ after plating. The Neu3 siRNA consists of four siRNA sequences: GCAGAGAUGCGUACCUCAA, CCAACAACUCUGCGAGCCU, CCAAACAAAUUCCGAGCAG, and GGACAGGGCUUGUUCGCGU (ON-TARGETplus SMART pool rat Neu3 siRNA, 117185, Thermo Scientific). siGLO RISC-Free siRNA (Thermo Scientific) was used as a negative control and transfection indicator. Briefly, DharmaFECT 3 (Thermo Scientific) and siRNA were diluted separately in serum-free DMEM supplemented with $1 \%$ insulin-transferrin-selenium and $10 \mathrm{ng} / \mathrm{ml} \mathrm{NGF}$ before mixing together and incubating with the DRGs (100 nM; $4 \mathrm{~h}$ ) and then replacing with normal medium.

\section{RNA purification and RT-PCR}

Dissociated DRGs were lysed and collected as per the manufacturer's instructions (PureLink RNA Mini Kit, Invitrogen) before being homogenized (Ultra-Turrax T8 homogenizer, IKA-Werke). RNA purification was performed before reverse transcription. The purified RNA was then reverse-transcribed using the SuperScript III First-Strand Synthesis SuperMix (Invitrogen). The cDNA synthesis reaction was followed by PCR with the following primers: Neu3, forward, 5'-CAGCTGGGATAGCAGAGGTC-3'; reverse, 5' -GAGTCCTGAAGCAAGCCAAC-3' , resulting in a 209 bp fragment; Neu4, forward, 5'-CCTGACCCTAGGACGAACAG-3'; reverse, 5' -GATGTGCGTGGTGATCAGAG-3', resulting in a $179 \mathrm{bp}$ fragment. The PCR was run at 26,29 , and 32 cycles separately and imaged using agarose gel electrophoresis.

\section{Immunocytochemical staining of cultures}

Both DRG and retina explant cultures were fixed with 4\% PFA for 10 min and washed with PBS before immunostaining. To analyze Neu3 sialidase activity, we calculated the relative levels of GDla (substrate) and GM1 (product) on individual axonal membranes rather than absolute mean values of GD1a and GM1 ganglioside. A mean of 30-60 axons per chamber ( 2 DRGs/chamber) was considered as $n=1$ /experimental condition. Fixed cultures were incubated with one of the following antibodies: rabbit anti-Neu3 sialidase/PMGS (Rodriguez et al., 2001), mouse anti-GD1a ganglioside (kind gift from Prof. Ronald Schnaar, Johns Hopkins University, Baltimore, MD), mouse anti-GDla ganglioside and mouse antiGT1b ganglioside (Merck Millipore) or stained using recombinant cholera toxin-B subunit (CTB) conjugated to AlexaFluor-555 (Invitrogen), and mouse anti $\beta 3$-tubulin. To analyze the signaling cascade regulating Neu3 sialidase activity, DRG and retinal cultures were analyzed with the following primary antibodies: mouse anti-P38MAPK, rabbit anti-phospho P38MAPK, and rabbit anti-ERK and mouse anti-phospho ERK (Cell Signaling Technology). Subsequently, cultures were washed in PBS and incubated with the following fluorescent secondary antibodies: 
anti-rabbit AlexaFluor-660, and anti-mouse or anti-rabbit AlexaFluor488 (Invitrogen) before being mounted onto slides using Fluorosave (Calbiochem).

\section{Surgeries}

All surgeries were performed in accordance with the United Kingdom Animals (Scientific Procedures) Act of 1986 and United Kingdom Home Office regulations. Adult (2- to 3-month-old) male Sprague Dawley rats were used for all in vivo experiments. Animals were kept in $12 \mathrm{~h} \mathrm{light/dark}$ exposure, and food and water was provided ad libitum.

Animals were anesthetized using 1-2\% isoflurane in a mixture of $50 \%$ nitrous oxide and $50 \%$ oxygen for sciatic nerve surgery. The right sciatic nerve was exposed and crushed proximal to its division into tibial and common peroneal nerves with Dumont \#5 forceps, crushing twice for 10 s duration each.

Optic nerve crushes were performed under ketamine:xylazine $(2: 1)$ anesthesia at a dosage of $0.1 \mathrm{ml} / 100 \mathrm{~g}$. The conjunctiva of the right optic nerve was cut laterally near the cornea, and the retractor bulbar muscle was separated. The optic nerve was exposed and cleared of fascial coverings and crushed twice for $10 \mathrm{~s}$ duration each time.

\section{Immunohistochemistry}

Animals were euthanized with an overdose of Euthatal (sodium pentobarbitol) at different time points after injury (2, 6, and $12 \mathrm{~h}$ ) and transcardially perfused with $\mathrm{PBS}, \mathrm{pH} 7.4$, and $4 \% \mathrm{PFA}, \mathrm{pH}$ 7.4. A 2-cm-long segment of sciatic or optic nerve centered around the crush site (or contralateral control nerves) was removed and postfixed overnight in $4 \%$ PFA before cryoprotecting in $30 \%$ sucrose in PBS, pH 7.4. Tissue was embedded in OCT (RA Lamb) and sectioned longitudinally at $12 \mu \mathrm{m}$ (sciatic nerve) or at $10 \mu \mathrm{m}$ (optic nerve) thickness in a cryostat and mounted on slides (Superfrost Plus; VWR).

Sections were permeabilized in $0.2 \%$ saponin in PBS and blocked with $10 \%$ NGS and 2\% BSA in PBS before incubating with one of the following antibodies: rabbit anti neurofilament-heavy chain (sciatic nerve) or rabbit anti- $\beta$ III tubulin, mouse anti-GD1a, CTB (for GM1 ganglioside), and anti-S100 $\beta$. Sections were rinsed in PBS-Saponin before incubating with appropriate secondary antibodies, and coverslipping with Fluorosave.

\section{Quantification of immunofluorescence}

In vitro. Photographs of cut axons were captured on a Leica DM6000B epifluorescent microscope equipped with a Leica DFC 350FX digital camera (Leica) using a $40 \times$ objective lens $(40 \times / 1.00-0.50$ Oil). Image acquisition using Leica Application Suite used identical exposure times and microscope settings. Quantification of images was performed using NIH ImageJ software.

In vivo. Photographs of sciatic (SN) and optic nerve (ON) sections adjacent to the crush site were captured using a Leica TCS SP2 confocal microscope (Leica) using $40 \times(40 \times / 1.15$ Oil CS) (for SN) or $63 \times(63 \times /$ 1.30 Oil CS) (for ON) magnification. Image acquisition using Leica Application Suite used identical exposure times and microscope settings. Image quantification was performed using confocal software using optical sections ( $0.800 \mu \mathrm{m}$ thick) to analyze the levels of GM1 and GD1a gangliosides parallel and between axonal and Schwann cell markers. Areas of interest were randomly chosen based on areas adjacent and parallel to NF-H-positive staining from a single confocal image. Intensities were measured as ratio of CTB (for GM1 ganglioside):GDla ganglioside normalized against background. In experiments where CTB or Neu3 sialidase intensity was measured, normalization was performed using intensity of an axonal marker, $\beta$ III-tubulin. To ensure that intensity differences were not due to differences in axon size, intensity measurements were normalized for the area of individual axon. Images were processed with Adobe Photoshop CS4 and assembled in Adobe Illustrator CS4.

\section{Statistical analysis}

Data were first analyzed for normality using Shapiro-Wilk normality test (Origin version 8; Originlab). $p$ values of all data were above set $\alpha$ levels of 0.05 and therefore considered to be normally distributed. Results from the analyses were expressed as mean \pm SEM. Statistical analysis was performed using Prism version 5 (Graphpad). For all experiments, Student's $t$ test (two-tailed), one-way ANOVA, and two-way ANOVA were applied as appropriate. Significant interactions were analyzed using paired $t$ tests and Bonferroni post hoc tests as appropriate.

\section{Results}

\section{Axotomy leads to an increase in the ratio of GM1/GD1a gangliosides due to Neu3 sialidase activation}

As a model of the first steps in successful axon regeneration, we used regeneration of growth cones in adult DRG axons grown on PDL/laminin. These axons regenerate a new growth cone and begin to elongate within $30 \mathrm{~min}$ after axotomy (Chierzi et al., 2005). We investigated levels of GM1 and GD1a gangliosides on these axons and asked whether their relative levels change after axotomy and during successful neurite regeneration. Both DRG explant and dissociated cultures were fixed at $15 \mathrm{~min}$ or $1 \mathrm{~h}$ after axotomy and stained with CTB (marker for GM1 ganglioside, which we use throughout the paper to label GM1) and anti-GD1a ganglioside antibody (Fig. 1). Axotomy led to conversion of most of the GD1a to GM1, the ratio of GM1/GD1a having doubled within $15 \mathrm{~min}$ and returned back to uncut levels by $1 \mathrm{~h}$ (Fig. $1 A-C, G, H-J, N)$. The change in the ratio was observed along the entire length of axons in our field of observation (200-300 $\mu \mathrm{m}$ from the proximal end of cut axons). Results are expressed as a ratio between GM1 and GD1a because absolute levels in individual axons vary depending on axon size while the ratio varies little, serving as a more representative indicator of local Neu3 sialidase activity on axons. The overall ganglioside level, measured as GD1a + GM1, did not change significantly after axotomy or after other our interventions, except for inhibition of ganglioside synthesis (data not shown). Because desialylation of GDla to produce GM1 on the cell surface is usually mediated by Neu3 sialidase, we confirmed the presence of Neu3 sialidase on DRG axons by immunostaining using an antibody described previously (Rodriguez et al., 2001) (Fig. 1V). Axons were immunolabeled without permeabilization demonstrating the presence of Neu3 sialidase on the axonal surface, which could be removed by inhibition of cholesterol synthesis with lovastatin or using methyl $\beta$ cyclodextrin (data not shown), additionally confirming its presence in cholesterol-rich domains (Kopitz et al., 1996; Kalka et al., 2001; Miyagi et al., 2008a,b). To verify that conversion of GD1a to GM1 is due to the action Neu3 sialidase, we blocked its activity in DRG explant cultures using the specific sialidase inhibitor, NeuAc2en (Rodriguez et al., 2001), or in dissociated DRG cultures with siRNA for NEU3, the gene that generates Neu3 sialidase. The increase in GM1 after axotomy was greatly reduced by NeuAc2en treatment; there was only a small change in the GM1/GD1a ratio after axotomy (Fig. 1D-G). Using RNA interference for NEU3, we further confirmed that a reduction in Neu3 sialidase prevented the change in the GM1/GD1a ratio after axotomy (Fig. $1 K-N$; controls described below). Furthermore, as $\mathrm{Neu} 3$ sialidase acts to convert many gangliosides, including GD1a, GT1b, GD1b, and GQ1b to GM1, we investigated whether GT1b was also affected by axotomy. Comparable to GD1a, axotomy led to conversion of GT1b to GM1, the ratio of GM1/GT1b having almost tripled within $15 \mathrm{~min}$ and decreasing back near uncut levels by $1 \mathrm{~h}$ (Fig. 1O-Q,U). After siRNA knockdown of Neu3, the GM1/GT1b ratio was unchanged after axotomy (Fig. $1 R-U)$. To determine whether axotomy increases GM1 conversion through Neu3 sialidase upregulation or by activation, we compared Neu3 sialidase protein levels on uncut axons and on 

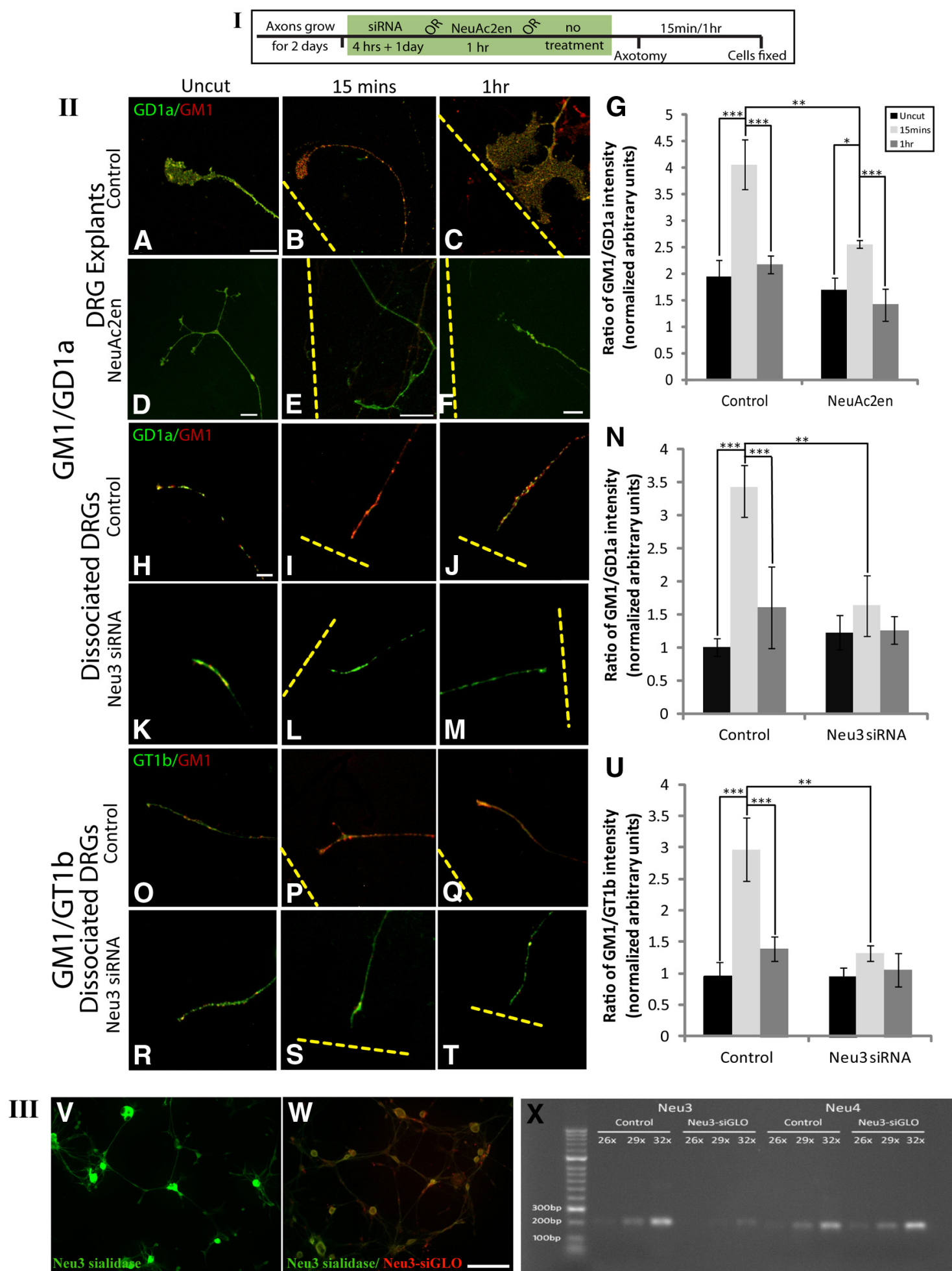

Figure 1. DRG axotomy causes a transient increase in the ratio of GM1/GD1a and GM1/GT1b gangliosides. I, Schematic of experimental design. II, Fluorescent images of adult DRG explants ( $\boldsymbol{A}-\boldsymbol{F}$ ) or dissociated DRGs $(\boldsymbol{H}-\boldsymbol{M}, \boldsymbol{O}-\boldsymbol{T})$ with axons uncut $(\boldsymbol{A}, \boldsymbol{D}, \boldsymbol{H}, \boldsymbol{K}, \boldsymbol{O}, \boldsymbol{R}), 15 \mathrm{~min}(\boldsymbol{B}, \boldsymbol{E}, \boldsymbol{I}, \boldsymbol{L}, \mathbf{O}, \boldsymbol{P}, \mathbf{S})$, or $1 \mathrm{~h}$ after axotomy $(\boldsymbol{C}, \boldsymbol{F}, \boldsymbol{J}, \boldsymbol{M}, \boldsymbol{Q}, \boldsymbol{T})$. DRGs are double-labeled for $G D 1$ a ganglioside (green) in $\boldsymbol{A}-\boldsymbol{F}$, $\boldsymbol{H}-\boldsymbol{M}$ (or GT1b ganglioside in $\mathbf{O}-\boldsymbol{T}$ ) and (TB for GM1 ganglioside (red) under normal conditions $(\boldsymbol{A}-\boldsymbol{C}, \boldsymbol{H}-\boldsymbol{J}, \mathbf{O}-\mathbf{Q})$, after exposure to NeuAc2en (D-F), or after Neu3 siRNA transfection ( $\boldsymbol{K}-\boldsymbol{M}, \boldsymbol{R}-\boldsymbol{T})$. In this and subsequent images, axotomy/nerve crush is indicated by dashed yellow lines, where applicable. GM1 and GD1a or GT1b ganglioside intensities were plotted ( \pm SEM) in $\mathbf{G}$ (DRG explants, $40-50$ axons/chamber, 2 DRG/chamber, $n \geq 4$ chambers) or $\boldsymbol{N}, \boldsymbol{U}$ (dissociated DRGs, 2 axons/chamber, $n=5$ ). III, Reduction of Neu3 expression with Neu3 siRNA. $\boldsymbol{V}, \boldsymbol{W}$, Fluorescent images of adult DRG neurons after immunocytochemistry for Neu3 sialidase, without siRNA transfection $(\boldsymbol{V})$, or after Neu3-siGL0 transfection $(\boldsymbol{W})$. Neurons transfected with Neu3-siGLO (red; $\boldsymbol{W}$ ) have reduced intensity for Neu3 sialidase staining (green). $X$, RT-PCR of siRNA-transfected adult DRG neurons, run at 26, 29, and 32 cycles. Neu3 siRNA is specific in knocking down the expression of Neu3 by $\sim 70 \%$ and has no effect on Neu4, a closely related sialidase. Controls are siGL0-transfected-only neurons (nonfunctional and nontargeting siRNA). ${ }^{*} p<0.05$ (one-way or two-way ANOVA, where appropriate). ${ }^{* *} p<0.01$ (one-way or two-way ANOVA, where appropriate). ${ }^{* *} p<0.001$ (one-way or two-way ANOVA, where appropriate). Scale bars: $\boldsymbol{A}$ for $\boldsymbol{A}-\boldsymbol{F}, 25 \mu \mathrm{m} ; \boldsymbol{H}$ for $\boldsymbol{H}-\boldsymbol{M}$ and $\boldsymbol{O}-\boldsymbol{T}$, $50 \mu \mathrm{m} ; \boldsymbol{V}-W, 250 \mu \mathrm{m}$ 

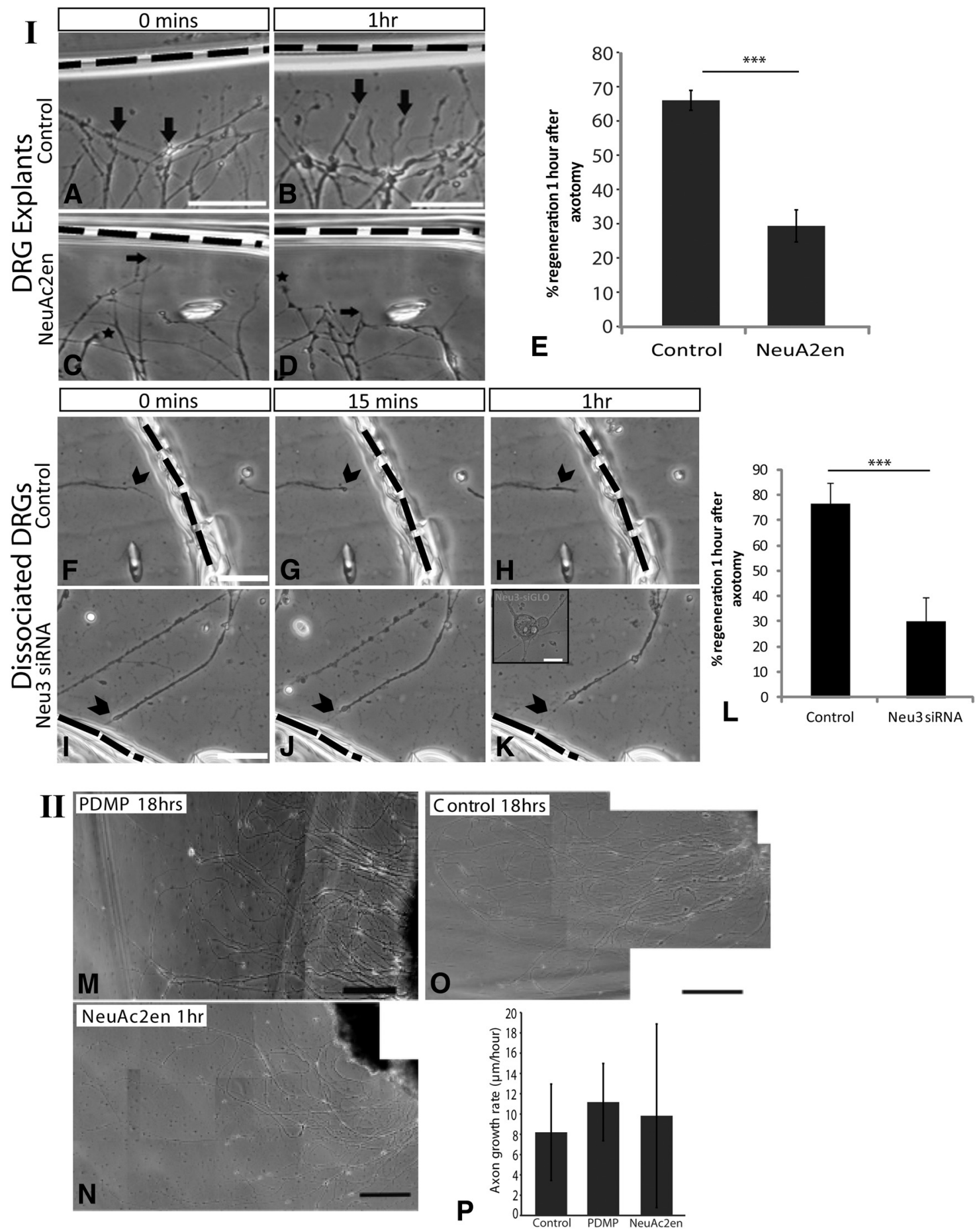

Figure 2. Reduction of Neu3 sialidase activity by the inhibitor, NeuAc2en, or by Neu3 siRNA transfection reduced regeneration after axotomy but not axon growth rate. $I$, Phasecontrast photomicrographs showing axons of DRG explants $(\boldsymbol{A}-\boldsymbol{D})$ or dissociated DRGs $(\boldsymbol{F}-\boldsymbol{K})$ immediately $(\boldsymbol{A}, \boldsymbol{C}, \boldsymbol{F}, \boldsymbol{I}), 15$ min (dissociated DRGs only; $\boldsymbol{G}, \boldsymbol{J})$, or $1 \mathrm{~h}$ after axotomy $(\boldsymbol{B}, \boldsymbol{D}, \boldsymbol{H}$, $\boldsymbol{K})$, under control conditions $(\boldsymbol{A}, \boldsymbol{B}, \boldsymbol{F}-\boldsymbol{H})$, and after exposure to NeuAc2en $(\boldsymbol{C}, \boldsymbol{D})$, or Neu3 siRNA transfection $(\boldsymbol{I}-\boldsymbol{K})$. Percentage regeneration $1 \mathrm{~h}$ after axotomy $( \pm$ SEM) plotted in $\boldsymbol{E}(n=$ 6 DRG explants) or after Neu3 siRNA transfection in $\boldsymbol{L}(2$ axons/chamber, $n=15$ chambers, dissociated DRGs). Arrows/arrowheads indicate axons that were cut, retracted at 15 min, and regenerated at 1 h. $\boldsymbol{C}, \boldsymbol{D}, \star A n$ uncut axon that continued to grow in the presence of NeuAc2en. $\boldsymbol{K}$, Inset, A merged phase-contrast and fluorescent image of the DRG cell body from $\boldsymbol{I}-\boldsymbol{K}$ demonstrating the presence of siGLO (red) confirming the siRNA uptake in the neuron. Controls $(\boldsymbol{F}-\boldsymbol{H})$ were performed with siGLO transfection only, or no treatment $(\boldsymbol{A}, \boldsymbol{B})$. II, $\boldsymbol{M}-\mathbf{O}$, Phase-contrast photo-montages of adult DRG axons $18 \mathrm{~h}$ after exposure to PDMP $(\boldsymbol{M}), 1 \mathrm{~h}$ after exposure to NeuAc2en $(\boldsymbol{N})$, and control ( $\boldsymbol{0})$. Photo-montages from similar areas were taken before addition of drugs and analyzed for growth rate in these as well as in corresponding control axons. Axon lengths were measured in drug-treated cultures and compared with corresponding control cultures to calculate the growth rate of axons (in $\mu \mathrm{m} / \mathrm{h} ; \pm$ SEM), which is plotted in $\boldsymbol{P}$ (each column represents a mean of $30-40$ axons from $n=3$ chambers/condition). ${ }^{* * *} p<0.001$ (two-tailed Student's $\boldsymbol{t}$ test). Scale bars: $\boldsymbol{A}-\boldsymbol{D}, 20 \mu \mathrm{m} ; \boldsymbol{F}-\boldsymbol{K}, 100 \mu \mathrm{m} ; \boldsymbol{K}$, inset, $50 \mu \mathrm{m} ; \boldsymbol{M}-\mathbf{0}, 200 \mu \mathrm{m}$. 


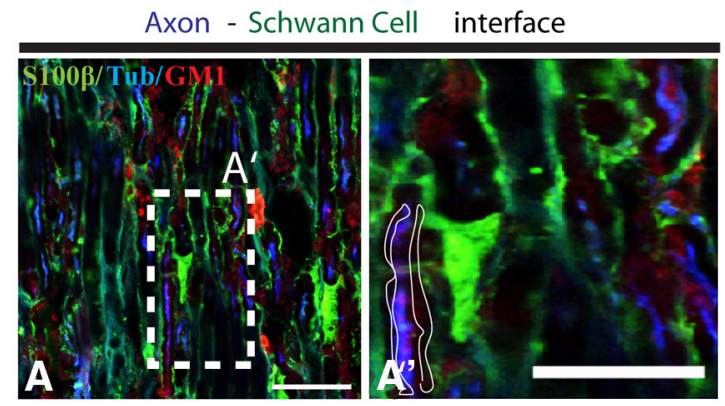

6hrs
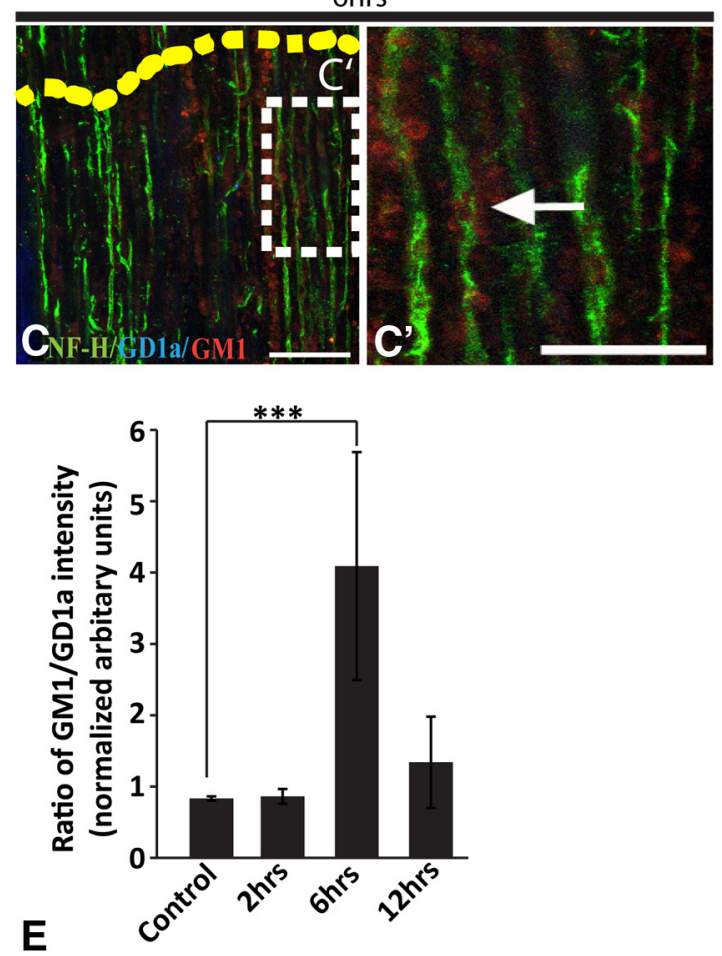

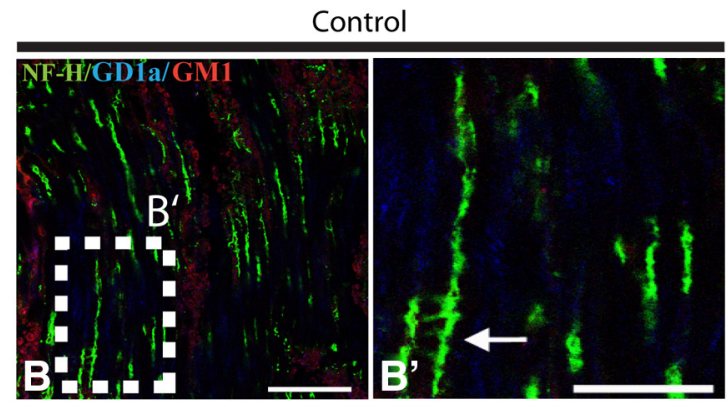

$12 \mathrm{hrs}$

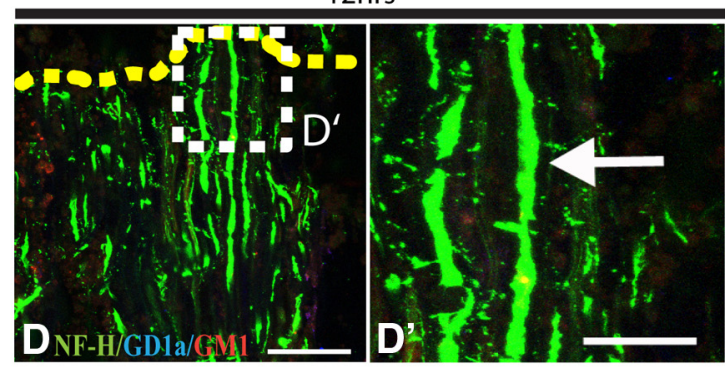

Figure 3. Neu3 sialidase is transiently activated in the sciatic nerve after crush injury. $\boldsymbol{A}, \boldsymbol{A}^{\prime}$, Confocal micrograph of sciatic nerve immunolabeled with S100 $\beta$ ( $\mathrm{green}$ ), CTB (red), and $\beta$ III-tubulin (blue). Area within dotted line $\left(\boldsymbol{A}^{\prime}\right)$ indicates representative regions parallel to axonal staining (blue) used to measure the intensities of GM1 and GD1a gangliosides. Schwann cell staining (green) performed to show its relative position to area of measurement. $\boldsymbol{B}-\boldsymbol{D}$, Fluorescent confocal images (longitudinal) of sciatic nerve uncrushed $\left(\boldsymbol{B}, \boldsymbol{B}^{\prime}\right), 6 \mathrm{~h}$ after crush $\left(6 \mathrm{~h} ; \boldsymbol{C}, \boldsymbol{C}^{\prime}\right)$, and $12 \mathrm{~h}$ after crush (12 h; $\left.\boldsymbol{D}, \boldsymbol{D}^{\prime}\right)$, triple-labeled for neurofilament-heavy chain (green), GD1a (blue), and GM1 gangliosides (CTB, red), with high-magnification images from dotted squares shown on right. Arrows indicate representative regions adjacent and parallel to axonal staining (NF-H-positive) used for measuring GM1 and GD1a ganglioside intensities. $\boldsymbol{E}$, The ratio of GM1/GD1a ganglioside intensities ( \pm SEM) over time after injury is plotted ( $n=6 /$ time point). ${ }^{* * *} p<0.001$ (one-way ANOVA). Scale bars: $\boldsymbol{A}-\boldsymbol{D}, 50 \mu \mathrm{m} ; \boldsymbol{A}^{\prime}-\boldsymbol{D}^{\prime}, 25 \mu \mathrm{m}$. Orientation of figures: top, caudal; bottom, rostral.

axons 15 min after injury by immunocytochemistry (Rodriguez et al., 2001; Da Silva et al., 2005). No significant change in Neu3 sialidase staining intensity was observed at 15 min after injury (data not shown), suggesting that an increase in Neu3 sialidase activity rather than an increase in expression or targeting to membrane was responsible for the acute rise in GM1/GD1a ratio after axotomy. These results indicate that reduction in Neu3 sialidase activity will reduce the level of desialytion of many gangliosides to GM1. However, to streamline our analyses for remaining experiments, we have chosen to assess changes in the ratio of GM1/GD1a intensity.

Knocking down the expression of Neu3 sialidase with siRNA does not affect the levels of closely related sialidases

To verify that the observed effect of knockdown of Neu3 on GM1 and GD1a and GT1b was specifically due to Neu3 sialidase, we evaluated expression levels of other related sialidases. After treatment with NEU3 siRNA, RT-PCR demonstrated a distinct reduction in Neu3 levels in dissociated DRG cultures, whereas levels of
Neu4 remained unaffected (Fig. $1 X$ ). Immunohistochemical staining for Neu3 sialidase in NEU3 siRNA-transfected cultures revealed decreases in Neu3 sialidase levels, indicating a reduction in Neu3 sialidase protein level compared with control cultures (Fig. $1 \mathrm{~V}, W$ ). As the NEU3 siRNA specifically reduced only Neu3, leaving Neu4 constant, our results suggest that changes in Neu3 sialidase activity are responsible for the conversion of GD1a (and GT1b) to GM1.

Inhibiting Neu3 sialidase activity reduces axon regeneration after axotomy

Because Neu3 sialidase activation converts more complex gangliosides to GM1 ganglioside and because GM1 has been shown to promote axonogenesis and axon growth, we hypothesized that inhibiting Neu3 sialidase's activity might inhibit regeneration in adult DRG axons. DRG explants (or dissociated DRG cultures) were grown for $48 \mathrm{~h}$ on PDL-laminin, exposed to NeuAc2en for $1 \mathrm{~h}$ (or siRNA transfection for $4 \mathrm{~h}$ followed by a $1 \mathrm{~d}$ incubation), axotomized, and regeneration was assessed at $15 \mathrm{~min}$ and $1 \mathrm{~h}$. 


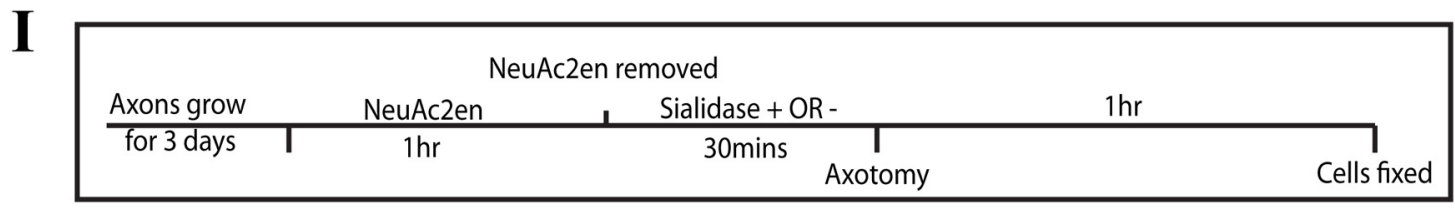

II
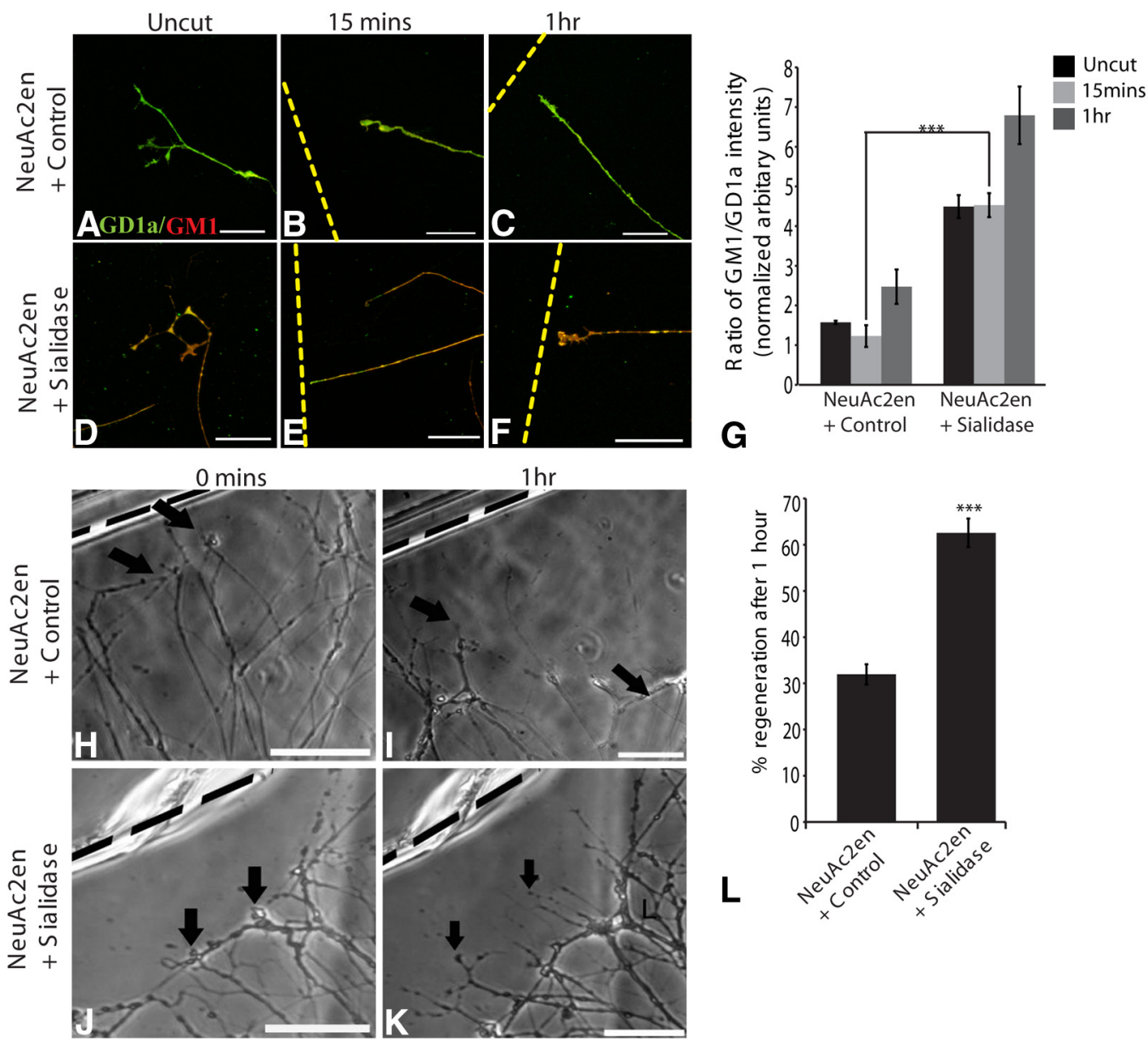

Figure 4. Exogenous sialidase rescues NeuAc2en-mediated inhibition of Neu3 sialidase activity and promotes axonal regeneration. $I$, Schematic of experimental design for sialidase rescue experiments in adult DRG axons. II, $\boldsymbol{A}-\boldsymbol{F}$, Fluorescent photomicrographs after exposure to Neu3 sialidase inhibitor (NeuAc2en) for $1 \mathrm{~h}$, which was removed and followed by no treatment (NeuAc2en + control; $\boldsymbol{A}-\boldsymbol{C}$ ) or exposure to exogenous Clostridium sialidase (NeuAc2en + Sialidase; $\boldsymbol{D}-\boldsymbol{F})$ with axotomy performed 30 min later showing adult DRG axons uncut $(\boldsymbol{A}, \boldsymbol{D}), 15$ min $(\boldsymbol{B}, \boldsymbol{E})$, and $1 \mathrm{~h}$ after axotomy $(\boldsymbol{C}, \boldsymbol{F})$, double-labeled for GD1a (green) and GM1 gangliosides (CTB, red). The ratio of GM1/GD1a gangliosides ( \pm SEM) was plotted in $\boldsymbol{G}$, with the GM1 and GD1a ganglioside intensity ratio normalized to background $(n \geq 4) .{ }^{* * *} p<0.001$ (two-way ANOVA). $\boldsymbol{H}-\boldsymbol{K}$, Phase-contrast photomicrographs showing DRG axons immediately after axotomy $(\boldsymbol{H}, \boldsymbol{J})$ and $1 \mathrm{~h}$ after exposure to NeuAc2en and removal (NeuAczen + control; $\boldsymbol{H}, \boldsymbol{I})$, or after exposure to NeuAc2en followed by exogenous (lostridium sialidase (NeuAc2en + Sialidase; $\boldsymbol{J}, \boldsymbol{K}$ ). Arrows indicate selected axons at axotomy and after $1 \mathrm{~h}$. Percentage regeneration ( \pm SEM) $1 \mathrm{~h}$ after axotomy plotted in $\boldsymbol{L}(n=6)$. ${ }^{* * *} p<0.001$ (Student's two-tailed $t$ test). Scale bars: $\boldsymbol{A}-\boldsymbol{F}, 50 \mu \mathrm{m} ;$ and $\boldsymbol{H}-\boldsymbol{K}, 30 \mu \mathrm{m}$.

Compared with untreated cultures (Fig. $2 A, B, F-H$ ), NeuAc2entreated DRG explants (Fig. 2C,D) and Neu3 siRNA-transfected dissociated DRGs (Fig. 2I-K) showed a marked reduction in regeneration (Fig. $2 E, L$ ). Axons were scored as regenerating if a growth cone was present at their tip and they had grown beyond their retraction point (Fig. $2 B, G$ ). Within dissociated cultures, retraction of axons in controls was most obvious within $15 \mathrm{~min}$ after axotomy, with regeneration occurring between $15 \mathrm{~min}$ and $1 \mathrm{~h}$. Uncut axons within the same cultures continued to grow in the presence of NeuAc2en with normal growth cone morphology (Fig. 2C,D). Additionally, we attenuated total ganglioside synthesis using two different inhibitors, fumonisinB1 and PDMP, and found that adult DRG axon regeneration was severely impaired (data not shown). To rule out toxicity from the ganglioside inhibitors, we measured the growth rate of uncut axons in the presence of PDMP or NeuAc2en and found no difference be- tween treated and control cultures over the same periods (Fig. $2 M-P$ ) with axon growth in uncut DRG cultures not affected by NeuAc2en (Fig. 2N). These results suggest that the GD1a to GM1 conversion mediated by rapid activation of Neu3 sialidase during the retraction period plays a significant role in the regeneration of cut DRG axons. However, high levels of GM1, or likewise reduced levels of complex gangliosides such as GD1a, are not needed for the continuing growth of mature axons.

\section{Sciatic nerve crush leads to GD1a to GM1 conversion in vivo}

We next investigated whether the conversion of GD1a to GM1 after axotomy occurs in the PNS in vivo. Sciatic nerve crushes were performed in adult rats, and the GM1/GD1a ganglioside ratio was measured immunohistochemically. To visualize changes in the vicinity of axon surfaces, we measured the intensity of GDla and GM1 in single optical sections, selecting narrow areas of interest 


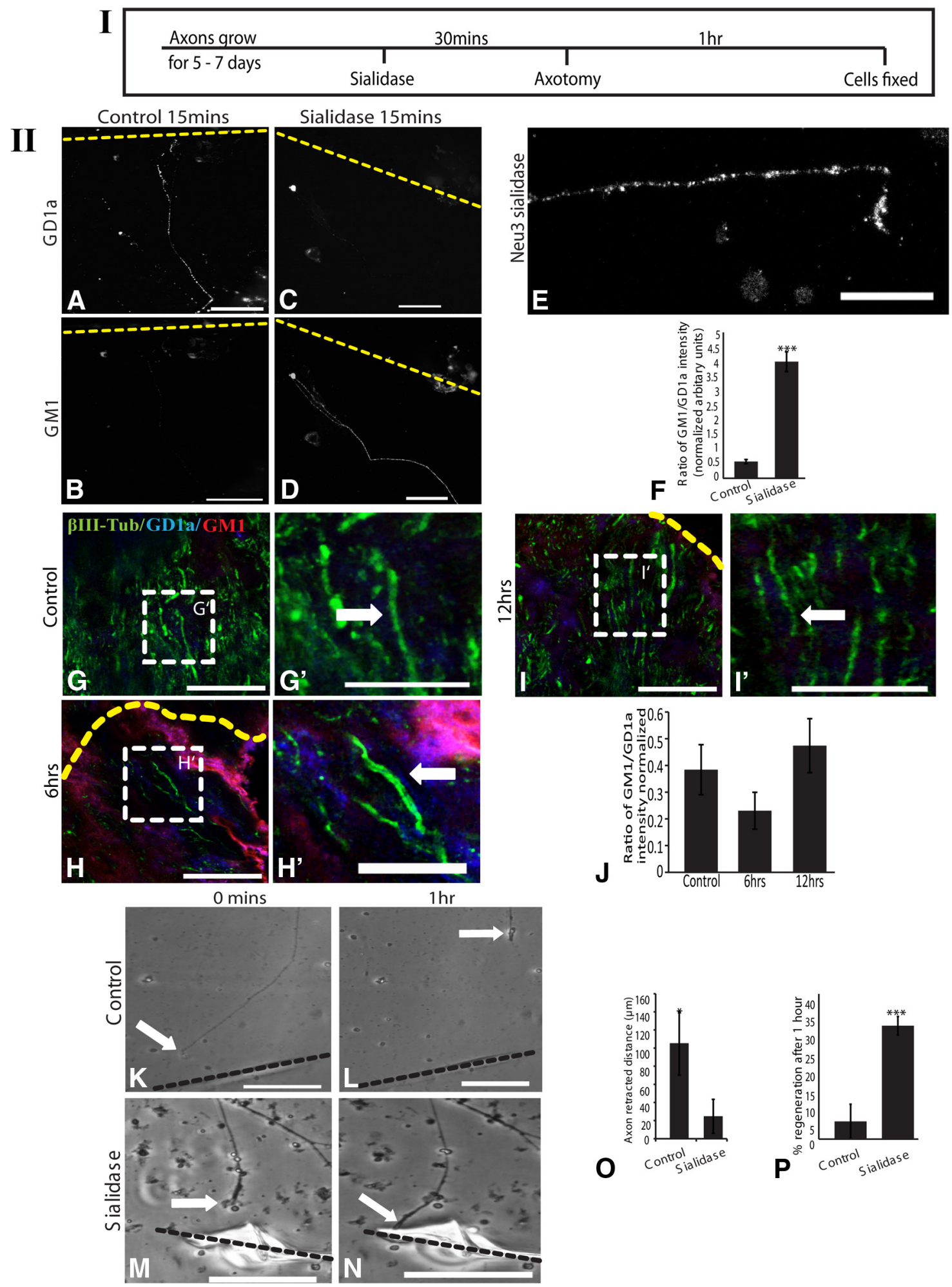

Figure 5. Neu3 sialidase remains inactivated after retinal axonal injury; however, exogenous sialidase can promote retinal axon regeneration. $I$, Schematic of timeline for retinal axon experiments. II, $\boldsymbol{A}-\boldsymbol{D}$, Fluorescent photomicrographs of adult retinal axons $15 \mathrm{~min}$ after axotomy. Axons are double-labeled for GD1a $(\boldsymbol{A}, \boldsymbol{C})$ and GM1 gangliosides (CTB; $\boldsymbol{B}, \boldsymbol{D})$ under normal conditions (control $15 \mathrm{~min} ; \boldsymbol{A}, \boldsymbol{B}$ ) or after exposure to Clostridium sialidase (sialidase $15 \mathrm{~min} ; \boldsymbol{C}, \boldsymbol{D}$ ). $\boldsymbol{E}$, Fluorescent photomicrograph showing Neu3 sialidase enzyme on an adult retinal axon. The ratio of GM1/GD1a ganglioside is plotted in $\boldsymbol{F}(30-40$ axons/chamber, $n \geq 6)$. $\mathbf{G}-\mathbf{I}$, Fluorescent confocal photomicrographs (longitudinal) of optic nerve uncrushed $\left(\boldsymbol{G}, \boldsymbol{G}^{\prime}\right), 6 \mathrm{~h}\left(\boldsymbol{H}, \boldsymbol{H}^{\prime}\right)$, and $12 \mathrm{~h}$ after crush $\left(I, I^{\prime}\right)$, triple-labeled for $\beta$ Ill-tubulin (green), GD1a (blue), and GM1 gangliosides (CTB, red). High-magnification images (') from the dotted squares in the corresponding pictures denote representative areas used for GD1a and GM1 quantification. Arrows indicate regions parallel to axonal staining used for measuring intensities of GM1 and GD1a gangliosides. The ratio of GM1/GD1a gangliosides ( \pm SEM) parallel to axonal staining before and after optic nerve injury and normalized against background intensity levels is plotted in $\boldsymbol{J}(n=3)$. $p$ values by one-way ANOVA. $\boldsymbol{K}-\boldsymbol{N}$, Phase-contrast photomicrographs showing retinal axons at time of axotomy $(0 \mathrm{~min} ; \boldsymbol{K}, \boldsymbol{M})$ and $1 \mathrm{~h}$ after axotomy $(1 \mathrm{~h} ; \boldsymbol{L}, \boldsymbol{N})$, retracting under normal conditions (control; $\boldsymbol{K}, \boldsymbol{L})$, or regenerating after exposure to Clostridium sialidase applied $30 \mathrm{~min}$ before axotomy (sialidase; $\boldsymbol{M}, \boldsymbol{N}$ ). Plots of mean distance of axon tip retraction from site of injury measured $1 \mathrm{~h}$ after injury in the presence or absence of exogenous sialidase $(n \geq 6)(\boldsymbol{O})$ and percentage of axons that regenerated past retraction position in retinal axons $1 \mathrm{~h}$ after injury $(n \geq 6)(\boldsymbol{P}) \pm S E M$. ${ }^{* * *} p<0.001 ;{ }^{*} p<0.05(S t u d e n t ' s t$ test, two-tailed). Scale bars: $\mathbf{A}-\boldsymbol{D}, 100 \mu \mathrm{m} ; \boldsymbol{E}, \boldsymbol{G}^{\prime}-\boldsymbol{I}^{\prime}, 25 \mu \mathrm{m} ; \mathbf{G}-\mathbf{I}, \boldsymbol{K}-\mathbf{N}, 50 \mu \mathrm{m}$. 

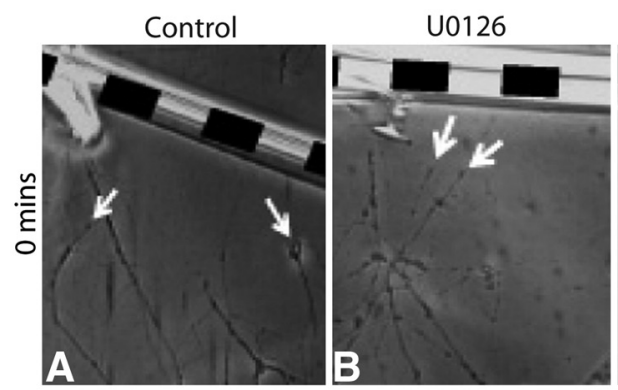

w/o Calcium
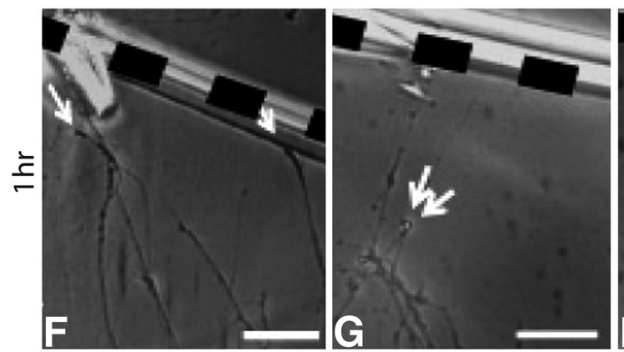
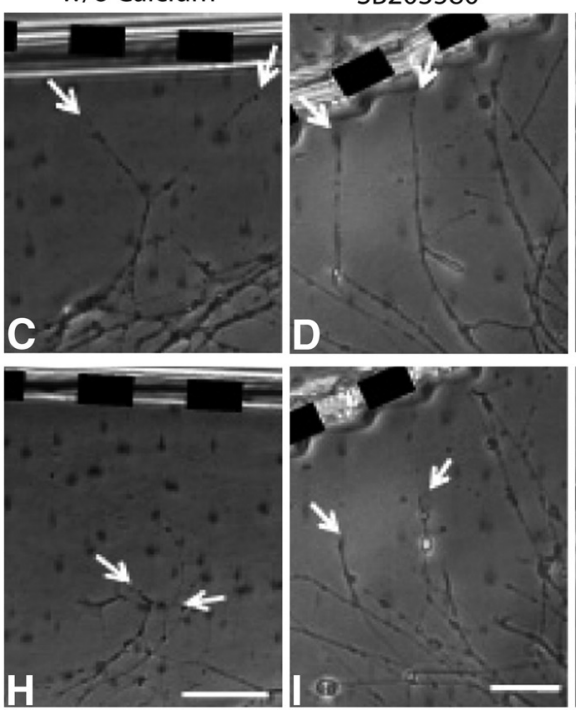

SB203580

Rapamycin
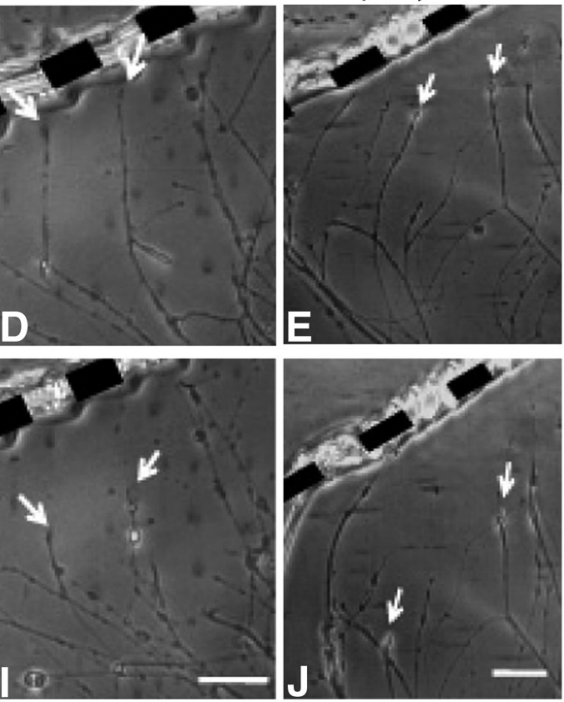

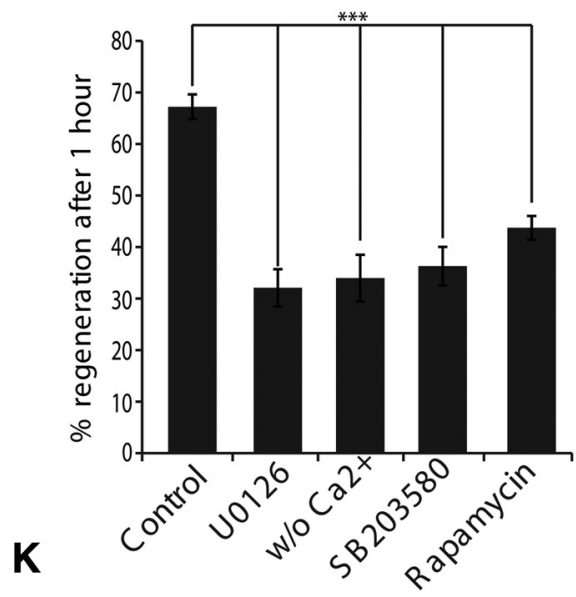

Figure 6. Axon regeneration is regulated by $\mathrm{eCa}^{2+}, \mathrm{P38MAPK}, \mathrm{ERK}$, and mTOR signaling cascades. $A-J$, Phase-contrast photomicrographs of adult DRG axons photographed immediately after axotomy $(\boldsymbol{A}-\boldsymbol{E})$ and $1 \mathrm{~h}$ after axotomy $(\boldsymbol{F}-\boldsymbol{J})$, under normal conditions (control; $\boldsymbol{A}, \boldsymbol{F})$ and after exposure to inhibitors of ERK $(\mathrm{U} 0126 ; \boldsymbol{B}, \boldsymbol{G})$, removal of $\mathrm{Ca}^{2+}$ (without calcium; $\left.\boldsymbol{C}, \boldsymbol{H}\right)$, inhibitors of P38MAPK (SB203580; $\boldsymbol{D}, \boldsymbol{I})$, and mTOR (rapamycin; $\boldsymbol{E}, \boldsymbol{J}$ ). Inhibition of all these pathways attenuated the ability of axons to regenerate. Percentage regeneration of axons ( \pm SEM) is plotted in $\boldsymbol{K}$ $(n \geq 5)$. ${ }^{* *} p<0.001$ (one-way ANOVA). Scale bars, $50 \mu \mathrm{m}$.

parallel to axons in between $\mathrm{S} 100 \beta$-stained Schwann cells and $\beta$ IIItubulin or neurofilament stained axonal profiles (Fig. $3 A^{\prime}$ ). Normal adult sciatic nerve had a very low ratio of GM1/GD1a (Fig. $3 B, B^{\prime}$ ). However, this ratio changed after nerve crush resulting in a fourfold increase within $6 \mathrm{~h}$ after crush injury, returning back to uncrushed levels by $12 \mathrm{~h}$ (Fig. 3B-E). Because axons and Schwann cells are in direct contact, we cannot rule out that some of the changes in sciatic nerve occurred on Schwann cells, but our in vivo results closely parallel changes we observed on axons in vitro, albeit with a longer time course.

\section{Exogenous sialidase rescues NeuAc2en-mediated inhibition} of Neu3 sialidase activity and promotes neurite regeneration To confirm the requirement for sialidase action in axon regeneration and to control for nonspecific effects of NeuAc2en, a rescue experiment was performed in cultured DRGs. After inhibiting endogenous Neu3 sialidase activity, we treated the cultures with exogenous sialidase to convert axonal gangliosides to GM1 (NeuAc2en + sialidase). First, a control experiment demonstrated that the effects of NeuAc2en inhibition on axon regeneration persist for at least $1 \mathrm{~h}$ after removal. Axotomy $30 \mathrm{~min}$ after removing NeuAc2en (NeuAc2en + control) showed that the inhibitory effect of NeuAc2en on Neu3 sialidase activity persisted after its removal, as indicated by a lack of increase in the GM1/ GD1a ratio (Fig. $4 A-C, G$ ). The regenerative ability of axons 30 min after NeuAc2en removal remained low, similar to cultures in which the inhibitor was still present (Fig. $4 H, I, L$ ). The persistent reduction of Neu3 activity after NeuAc2en withdrawal is unexpected after removal of a soluble competitive inhibitor and suggests longer-term effects that influence the activity of Neu3 in the axonal membrane. In a previous study, this inhibitor caused changes in axonal membrane structures and the cytoskeleton that persisted after inhibitor removal (Da Silva et al., 2005).

Having confirmed the persistent blocking of Neu3 sialidase activity after NeuAc2en removal, we assessed whether exogenous Clostridium sialidase dissolved in the culture medium could convert GD1a to GM1. Application of sialidase increased the GM1/ GD1a ratio in uncut axons to the level found after axotomy, and axotomy after sialidase treatment did not further increase GM1 levels (Fig. 4D-G). Treatment of Neu3 sialidase-inhibited cultures with sialidase restored their regenerative ability near the levels observed in normal conditions (Fig. $4 J-L$ ). These results 

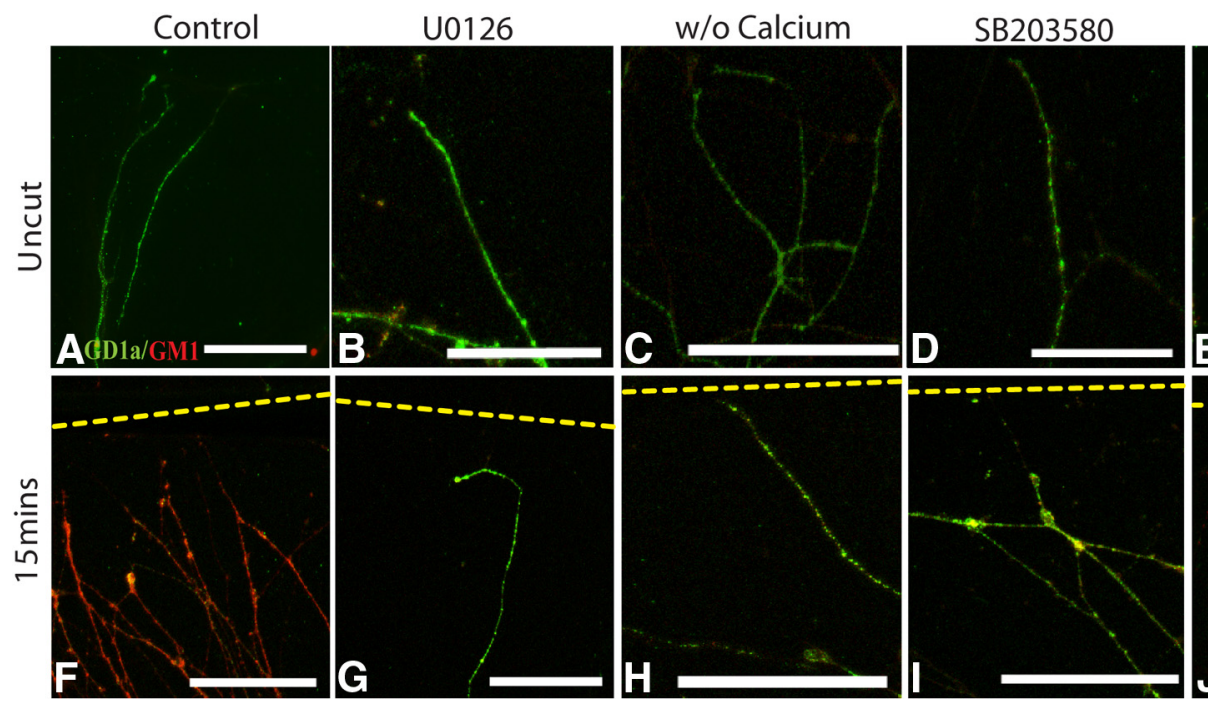

Rapamycin
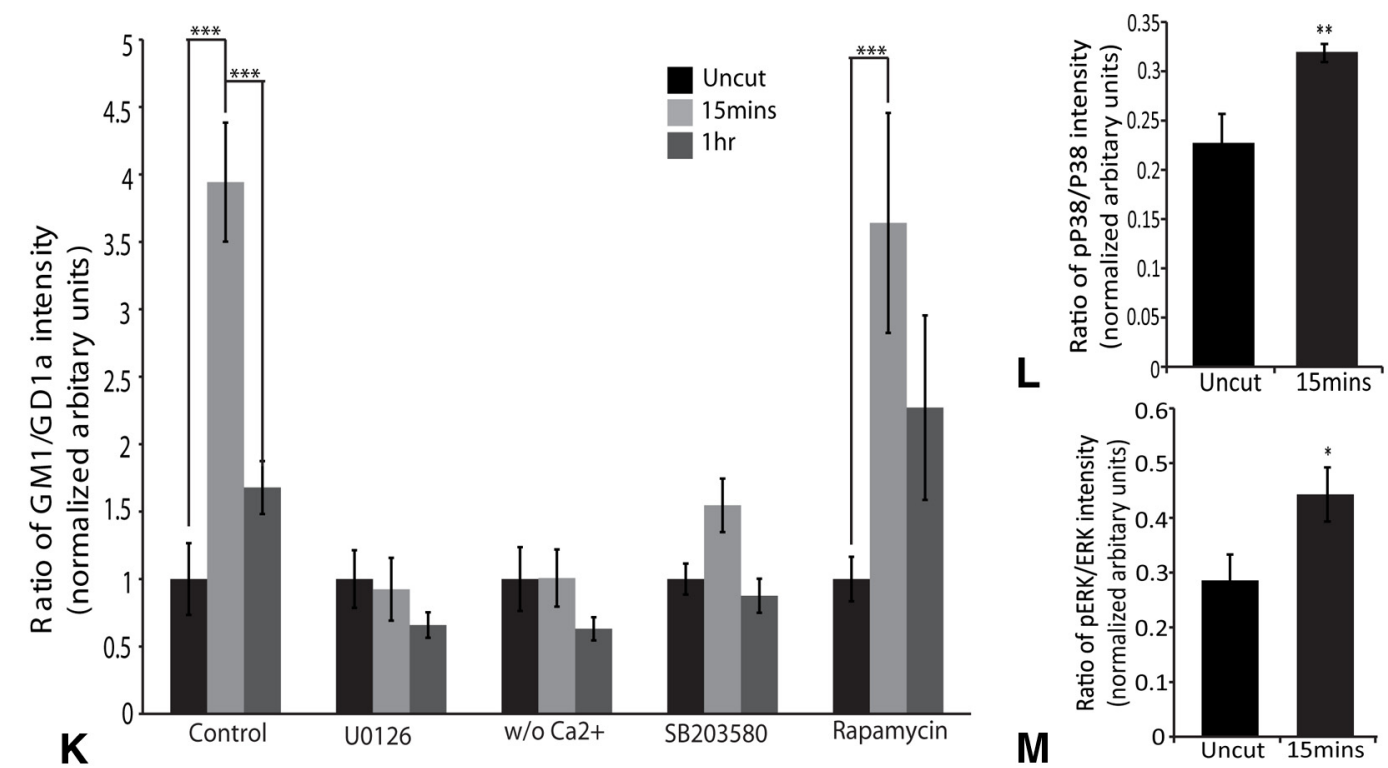

Figure 7. Neu3 sialidase activity is affected by $\mathrm{eCa}^{2+}{ }^{2+}, \mathrm{P} 38 \mathrm{MAPK}$, and ERK in adult DRG axons after axotomy. $\boldsymbol{A}-\boldsymbol{J}$, Fluorescent photomicrographs of adult DRG axons uncut $(\boldsymbol{A}-\boldsymbol{E})$ and 15 min after axotomy $(\boldsymbol{F}-\boldsymbol{J})$, double-labeled for GD1a (green) and GM1 gangliosides (CTB, red) under normal conditions (control; $\boldsymbol{A}, \boldsymbol{F}$ ) and after exposure to inhibitors of ERK (U0126; $\boldsymbol{B}, \boldsymbol{G})$, removal of $\mathrm{Ca}^{2+}$ (without calcium; $\boldsymbol{C}, \boldsymbol{H}$ ), inhibitors of P38MAPK (SB203580; $\boldsymbol{D}, \boldsymbol{I}$ ) or mTOR (rapamycin; $\boldsymbol{E}, \boldsymbol{J}$ ). The ratio of GM1/GD1a ganglioside intensities $( \pm$ SEM) normalized against background intensity levels is plotted in $\boldsymbol{K}$ ( $40-50$ axons/chamber, $2 \mathrm{DRGs} /$ chamber, $n \geq 3$ chambers). To avoid variability among uncut axons, axonal ratios of GM1/GD1a intensity levels were assigned the arbitrary value of 1 and GM1/GD1a levels at 15 min and $1 \mathrm{~h}$ after axotomy were normalized against corresponding uncut axons' ratio. $L$, $M$, The ratio of pP38MAPK/P38MAPK ( $L$; uncut, $n=4 ; 15$ min, $n=6)$ and pERK/ERK ( $M$; uncut, $n=4 ; 15 \mathrm{~min}, n=4$ ) uncut and 15 min after injury is plotted \pm SEM. ${ }^{* * *} p<0.001$ (two-way ANOVA). ${ }^{* *} p<0.01$ (Student's $t$ test, two-tailed). ${ }^{*} p<0.05$ (Student's $t$ test, two-tailed). Scale bars: $A-J, 50 \mu \mathrm{m}$.

suggest that GD1a to GM1 ganglioside conversion mediated either by Neu3 sialidase or by externally applied sialidase is important for regeneration of peripheral axons.

The Neu3 sialidase-mediated transient increase in the ratio of GM1/GD1 a ganglioside is not observed in retinal axons

In light of previous results, we asked whether the failure of regeneration of CNS axons might involve an inability to increase GM1 after axotomy. For these experiments, an axotomy was performed on axons extending from adult rat retinal explants. We have previously shown that only $5 \%$ of adult retinal axons regenerate after in vitro axotomy compared with $70 \%$ of adult DRG axons (Chierzi et al., 2005; Verma et al., 2005). The axons usually retract from the cut site and form an active retraction bulb, but fail to make a new growth cone. We found that adult retinal axons have a lower ratio of GM1/GD1a gangliosides on their surface than DRG axons. Additionally, there was no conversion of GDla to GM1 ganglioside 15 min after axotomy in retinal axons (Fig. $5 A, B$ ). To determine whether this was due to low levels of Neu3 sialidase in retinal axons, its abundance and distribution were assessed by immunofluorescence. Similar levels of staining and distribution of Neu3 sialidase to that found in DRG cultures were observed (Fig. 5E). To investigate whether the conversion of GDla to GM1 can occur in these axons in the presence of active sialidase, we applied exogenous sialidase to retinal cultures. The treatment induced high levels of GM1 ganglioside on retinal axons, bringing the GM1/GDla ratio close to that of axotomized or sialidase-treated adult DRG axons (Fig. 5C,D,F). These experiments suggest that Neu3 sialidase is present on adult retinal axons but is not activated by axotomy. 


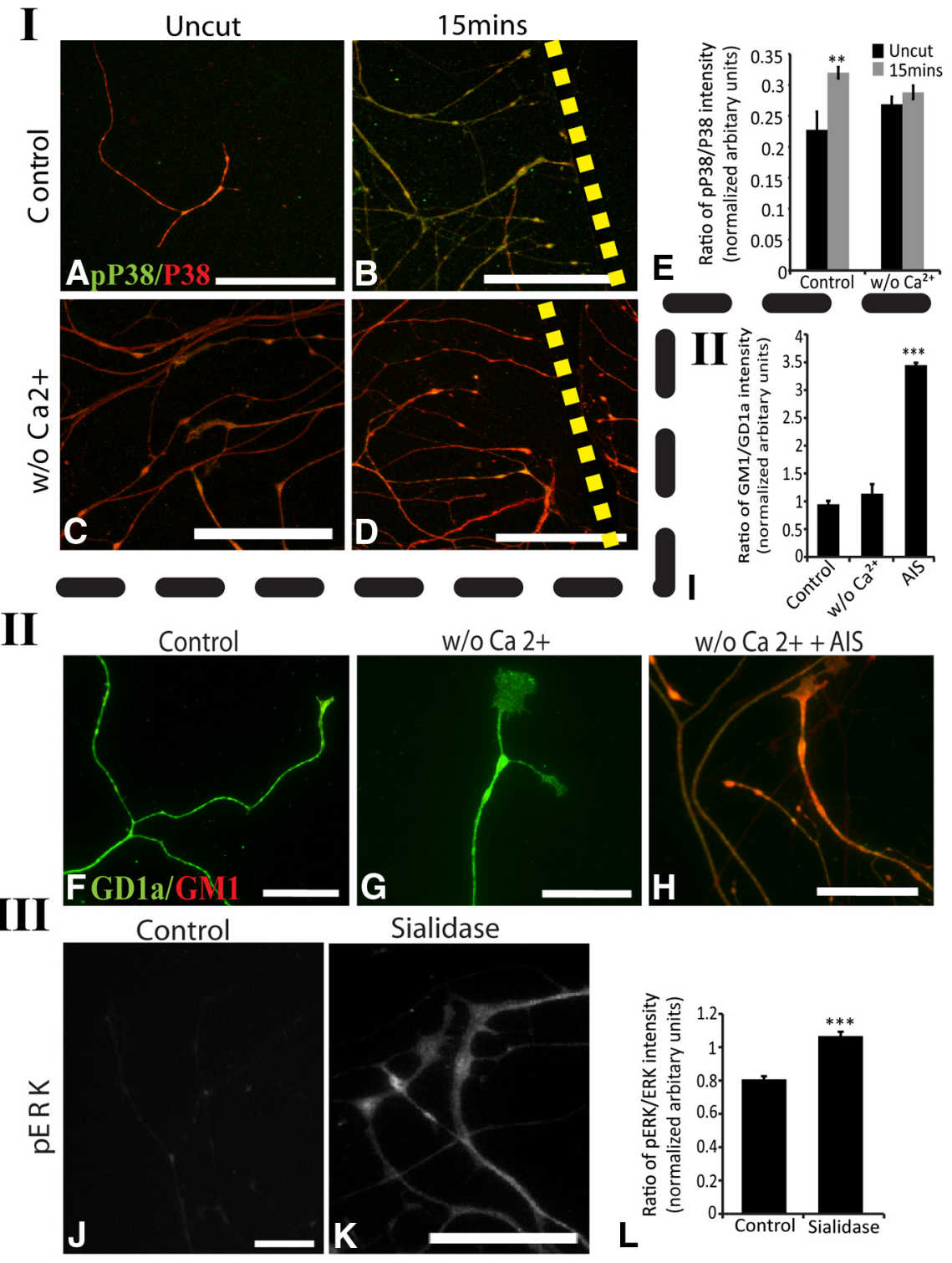

Figure 8. P38MAPK activation is the intermediary step between $\mathrm{eC}^{2+}$ influx and Neu3 sialidase activation after axonal injury, whereas ERK activation is downstream of Neu3 sialidase activation. $\boldsymbol{I}, \boldsymbol{A}-\boldsymbol{E}$, Fluorescent photomicrographs of adult DRG axons uncut $(\boldsymbol{A}, \boldsymbol{C})$ and 15 min after axotomy $(\boldsymbol{B}, \boldsymbol{D})$, double-labeled for P38MAPK (red) and phospho P38MAPK (green) under normal conditions (control; $\boldsymbol{A}, \boldsymbol{B}$ ) and after removal of $\mathrm{e}^{2+}{ }^{2+}$ (without $\left.\mathrm{Ca}^{2+} ; \boldsymbol{C}, \boldsymbol{D}\right)$. The ratio of phospho P38MAPK/P38MAPK intensities ( \pm SEM) normalized against background intensity levels is plotted in $\boldsymbol{E}$ (30-40 axons/chamber, 2 DRGs/chamber, $n=4-6$ chambers). II, Activation of P38MAPK using anisomycin increases Neu3 sialidase activity even in the absence of eCa ${ }^{2+} . \boldsymbol{F}-\boldsymbol{H}$, Fluorescent photomicrographs of uncut adult DRG axons, double-labeled for GD1a (green) and GM1 gangliosides (CTB, red) and under normal conditions (control; $\boldsymbol{F}$ ), after removal of eCa ${ }^{2+}$ (without $\mathrm{Ca}^{2+} ; \boldsymbol{G}$ ) and after exposure to anisomycin (AIS) in the absence of $\mathrm{eCa}^{2+}$ (without $\mathrm{Ca}^{2+}+\mathrm{AIS} ; \boldsymbol{H}$ ). The ratio of GM1/GD1a ganglioside intensities ( $\pm \mathrm{SEM}$ ) normalized against background intensity levels is plotted in I (30-40 axons/chamber, 2 DRGs/chamber, $n=4$ chambers). III, ERK activation is downstream of Neu3 sialidase activation. $J-L$, Fluorescent photomicrographs of adult uncut DRG axons, labeled for pERK under contro conditions $(\boldsymbol{J})$ and after exposure to exogenous sialidase $(\boldsymbol{K})$. The ratio of pERK/ERK intensities $( \pm \mathrm{SEM})$ normalized against background intensity levels is plotted in $\boldsymbol{L}\left(30-40\right.$ axons/chamber, containing 2 DRGs, $n=6$ chambers). ${ }^{* * *} p<0.001$ (Student's $t$ test, two-tailed). ${ }^{* *} p<0.01$ (Student's $t$ test, two-tailed). Scale bars, $50 \mu \mathrm{m}$.

To determine whether conversion of gangliosides by sialidase activity occurs after damage to the CNS in vivo, we performed an optic nerve crush injury in adult rats and measured GM1/GD1a levels in narrow regions adjacent to the axonal marker $\beta$ IIItubulin. No change in the GM1/GD1a ratio was observed in these axonal regions of the optic nerve after injury, and the ratio in undamaged nerves was similar to uncrushed sciatic nerve (Fig. $\left.5 G-I, G^{\prime}-I^{\prime}, J\right)$.
Treatment of retinal axons with sialidase increases their regeneration after axotomy

We next examined whether exogenous sialidase treatment enhances the ability of adult retinal axons to regenerate. After axotomy, retinal axons showed greater retraction than DRG axons, with many axons retracting out of the field of view (Fig. $5 \mathrm{~K}-\mathrm{O})$. Furthermore, only $4.8 \%$ of control axons regrew $1 \mathrm{~h}$ after axotomy beyond their point of initial retraction, whereas $21.3 \%$ of axons exposed to sialidase regenerated past their retraction point (Fig. 5P). These results demonstrate that conversion of more complex gangliosides to GM1 mediated by sialidase is an important component of the regenerative response and that exogenous sialidase treatment can enhance regeneration in adult retinal axons in vitro, although not to the level seen in sensory axons.

Neu3 sialidase activity is regulated by $\mathrm{eCa}^{2+}, \mathrm{ERK}$, and P38MAPK in adult DRG axons after axotomy

The preceding experiments indicate that axotomy activates Neu3 sialidase in adult DRG axons, but not in adult retinal axons, and that Neu3 sialidase activation is necessary for a successful regenerative response. We therefore investigated the signaling events that link axotomy to Neu3 sialidase activation and then to axon regeneration. Previous work has identified three signaling pathways (ERK, P38MAPK, and mTOR) (Agthong et al., 2009, 2012; Abe et al., 2010 ) as well as a local $\mathrm{Ca}^{2+}$ influx (Mandolesi et al., 2004; Cho and Cavalli, 2012; Cho et al., 2013), which occurs in response to axotomy and whose inhibition diminishes successful DRG axon regeneration (Chierzi et al., 2005; Verma et al., 2005). We confirmed that transient inhibition of any of these pathways with standard inhibitors attenuated the ability of adult DRG axons to regenerate (Fig. 6).

Next, we asked whether blocking these signaling pathways affected the conversion of GD1a to GM1 after axotomy. Removal of $\mathrm{eCa}^{2+}$, inhibition of ERK or P38MAPK prevented the axotomymediated increase in the GM1/GD1a ratio after axotomy, whereas blocking mTOR had no effect on the ratio (data shown in Fig. 7 legend). These results suggest that Neu3 sialidase activation in axotomized adult DRGs is regulated by a signaling cascade involving $\mathrm{eCa}^{2+}$, ERK, and P38MAPK.

Based on our results combined with knowledge of damage responses in other cell types where P38MAPK has been shown to be a stress response kinase (Coulthard et al., 2009), we hypothesized that, after axonal injury, the rapid influx of $\mathrm{Ca}^{2+}$ activates P38MAPK, which in turn leads to increased Neu3 sialidase activ- 


\section{ADULT RETINA}

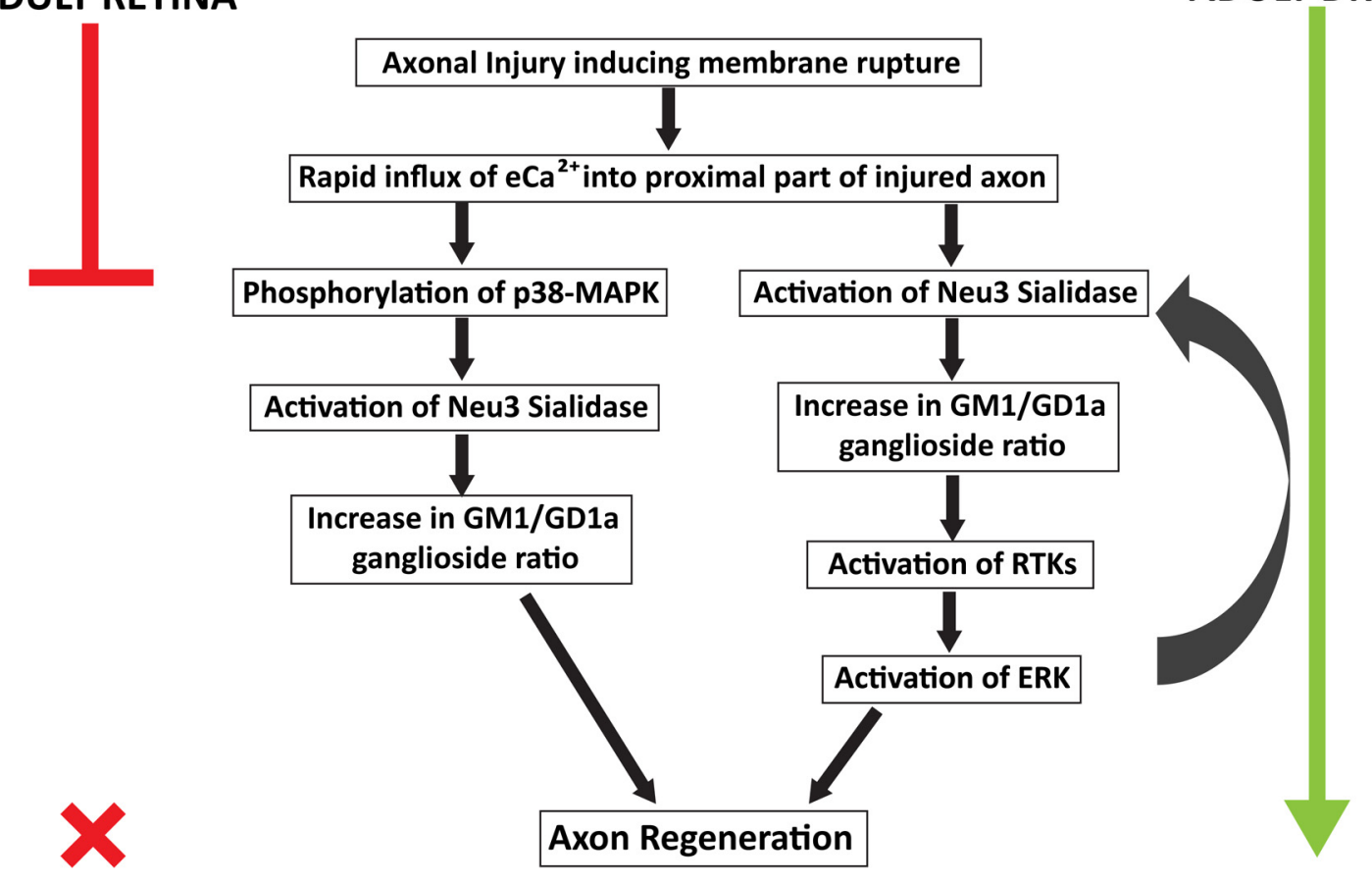

Figure 9. Diagram of proposed signaling cascade regulating Neu3 sialidase activity. Membrane rupture after axotomy leads to increased influx of eCa ${ }^{2+}$ into injured axons. Whereas in adult DRGs this results in either direct activation of Neu3 sialidase or an increased phosphorylation of P38MAPK resulting in increased Neu3 sialidase activation, this process is absent in adult retinal axons. Furthermore, ERK is activated downstream of Neu3 sialidase, which in turn further activates Neu3 sialidase through a positive feedback loop, a step attenuated in retinal axons, resulting in a two step block for successful Neu3 sialidase activity mediated regeneration.

ity. Based on previous work, Neu3 sialidase-mediated ganglioside conversion leads to increased signaling via the ERK pathway ( Da Silva et al., 2005). ERK activation acts on axonal growth mechanisms likely as part of a positive feedback loop leading to further activation of Neu3 sialidase (see Discussion and Fig. 9). We first investigated phosphorylation of P38MAPK (pP38MAPK) 15 min after axotomy of adult DRG axons in the presence or absence of $\mathrm{eCa}^{2+}$. In the absence of $\mathrm{eCa}^{2+}$, the increase in the pP38MAPK/P38MAPK ratio after axotomy was significantly reduced, suggesting that P38MAPK is downstream of $\mathrm{eCa}^{2+}$ entry (Fig. 8A-E). We next assessed whether P38MAPK activation could activate Neu3 sialidase. P38MAPK in uncut axons in the absence of $\mathrm{eCa}^{2+}$ was first activated by a common P38MAPK activator, anisomycin (Katoh-Semba et al., 2009; Mei et al., 2012), before the surface levels of GM1 and GD1a were measured. Anisomycin treatment led to a mild increase in pP38MAPK/P38MAPK, and this in turn resulted in a 3.5-fold increase in the ratio of GM1/GDla. Although anisomycin is not entirely specific to p38, coupled with the other results, the experiment suggests that Neu3 sialidase was activated by P38MAPK activation (Fig. 8F-I). Next, we examined whether ganglioside conversion can activate the ERK pathway, as demonstrated previously in embryonic neurons (Da Silva et al., 2005). To test this, we treated uncut adult DRG axons with exogenous sialidase to convert GD1a to GM1. This conversion resulted in an increased ratio of pERK/ERK (Fig. 8J-L). Together, these results support the proposed signaling events shown in Figure 9.

P38MAPK is not activated in adult retinal axons after axotomy

CNS axotomy has been shown to invoke a large calcium influx (Mandolesi et al., 2004); therefore, we hypothesized that the block in Neu3 sialidase activation in retinal axons might occur at the P38MAPK activation step. We evaluated whether the ratio of
pP38MAPK/P38MAPK changes 15 min after axotomy in retinal axons as observed in DRG axons. After axotomy, we did not observe an increase in the ratio of either pP38MAPK/P38MAPK or pERK/ERK levels (Fig. 10A-F). Therefore, we assessed whether Neu3 sialidase activation consequent on P38MAPK activation is operative in retinal axons. P38MAPK was activated using anisomycin, and we measured the GM1/GD1a ganglioside ratio. Anisomycin treatment led to a $100 \%$ increase in the levels of GM1/GD1a ganglioside (Fig. 10G-I).

Overall, our experiments indicate that, in DRG axons, Neu3 sialidase activation after axonal injury is triggered by influx of $\mathrm{eCa}^{2+}$ after membrane rupture, leading to activation of P38MAPK. However, in retinal axons, neither P38MAPK nor Neu3 sialidase is activated after axotomy. Active Neu3 sialidase or an externally applied sialidase changes the ratio of GM1/GD1a ganglioside, leading to ERK pathway activation and a successful regeneration response.

\section{Discussion}

Our study demonstrates that ganglioside composition on axons is important for growth cone production and that conversion of axonal gangliosides to GM1 by the axonal surface enzyme Neu3 sialidase is required for adult axon regeneration. CNS axons lack intrinsic regenerative ability, and our results strongly suggest that the lack of Neu3 sialidase activation after axotomy is a factor in poor regenerative ability. The importance of sialidase activity for CNS regeneration is supported by a previous study in which sialidase treatment after spinal cord injury promoted axon regeneration (Yang et al., 2006) and functional recovery (Mountney et al., 2010, 2013). Further work has shown that conversion of gangliosides to GM1 by Neu3 sialidase is critical during early neuronal differentiation for axonal specification (Da Silva et al., 2005; Abad-Rodriguez and Robotti, 2007). Furthermore, poor regen- 
I
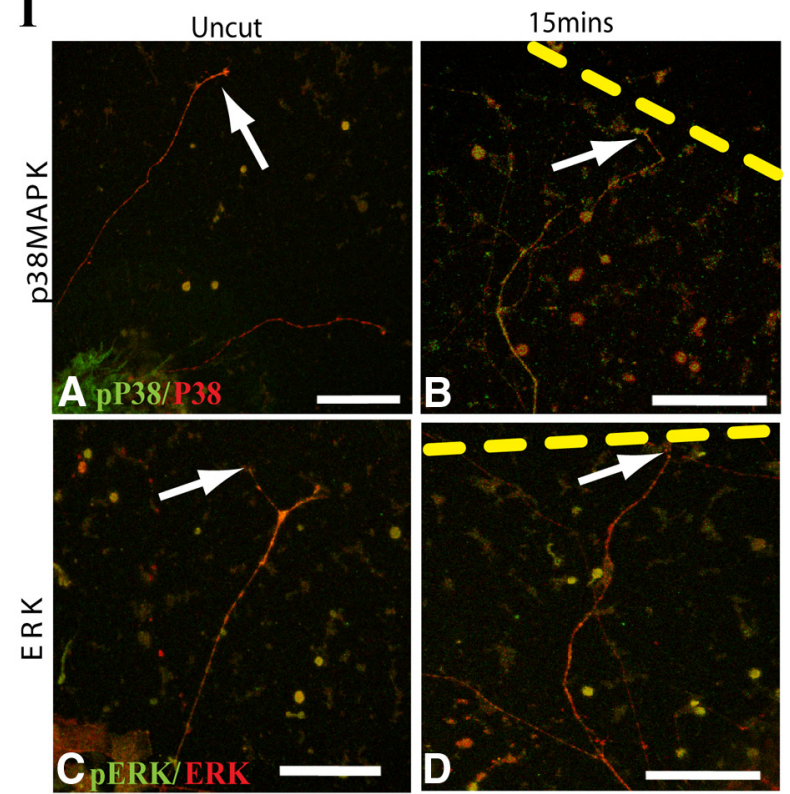

II

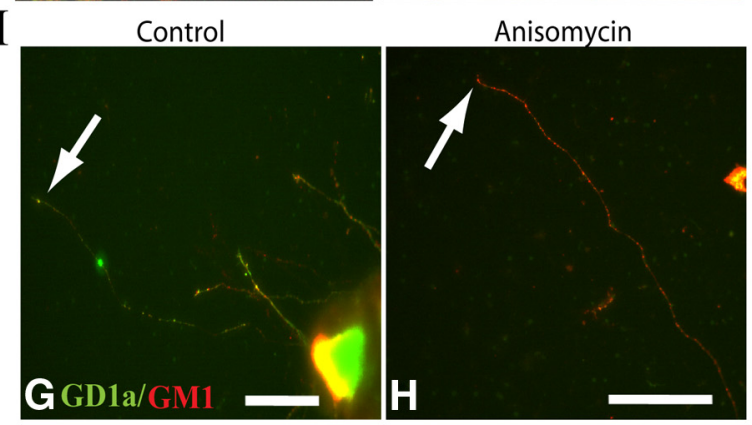

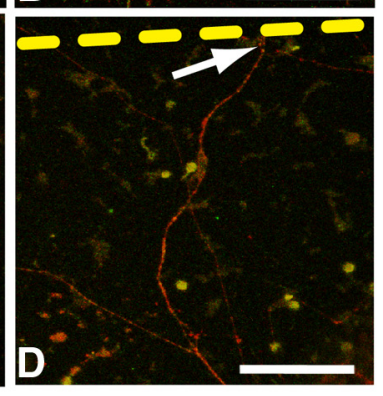

$\mathbf{F}$
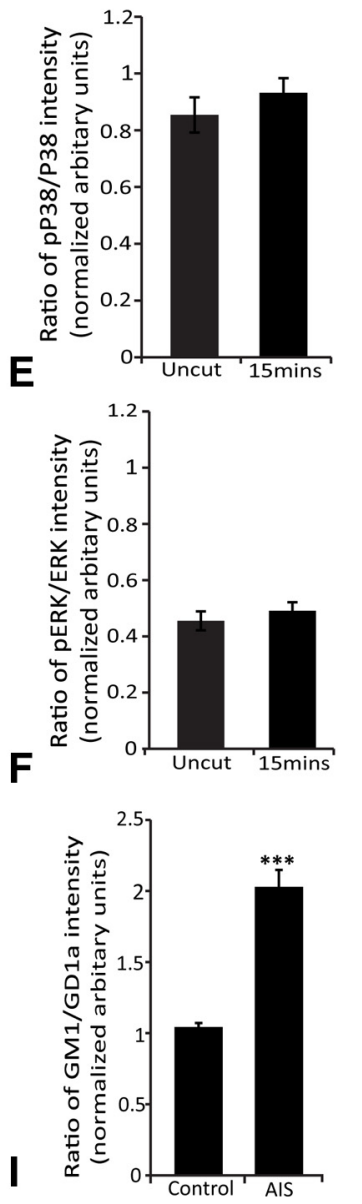

Figure 10. P38MAPK and ERK are not activated within 15 min after axotomy in retinal axons, but forced activation of P38MAPK increases Neu3 sialidase activity in retinal axons. $\boldsymbol{I}, \boldsymbol{A}-\boldsymbol{D}$, Fluorescent photomicrographs of adult retinal axons uncut $(\boldsymbol{A}, \boldsymbol{C})$ and 15 min after axotomy $(\boldsymbol{B}, \boldsymbol{D})$, double-labeled for P38MAPK $(\boldsymbol{A}, \boldsymbol{B})$ or ERK $(\boldsymbol{C}, \boldsymbol{D})$ (red) and pP38MAPK $(\boldsymbol{A}, \boldsymbol{B})$ or pERK $(\boldsymbol{C}, \boldsymbol{D})$ (green). Ratios of pP38MAPK/P38MAPK and pERK/ERK intensities ( \pm SEM) normalized against background intensity levels are plotted in $\boldsymbol{E}$ and $\boldsymbol{F}$ (30- 40 axons/chamber, $n=4$ chambers). $\boldsymbol{I I}, \mathbf{G}, \boldsymbol{H}$, Fluorescent photomicrographs of adult uncut retinal axons, double-labeled for GD1a (green) and GM1 gangliosides (CTB, red). Anisomycin increased the GM1/GD1a ratio (compare $\boldsymbol{H}$ with $\boldsymbol{G}$ ). $\boldsymbol{I}$, Ratios of GM1/GD1a ganglioside intensities ( \pm SEM) normalized against background intensity levels are plotted in I ( $30-40$ axons/ chamber, $n \geq 7$ ). ${ }^{* *} p<0.001$ (Student's $t$ test, 2-tailed). Scale bars, $50 \mu \mathrm{m}$.

eration of PNS axons has been observed in animals lacking complex gangliosides (Kittaka et al., 2008) and after treatment with antibodies to GM1 ganglioside (Lehmann et al., 2007; Lopez et al., 2010).

\section{Regulation of Neu3 sialidase activity}

Sialidases, such as Neu3, act to remove terminal sialic acid groups, converting gangliosides, such as the main CNS gangliosides GD1a, GD1b, and GT1b to GM1. After axotomy of DRG neurons, we observed a rapid increase in GM1 and a decrease in both GD1a and GT1b, which was prevented by Neu3 sialidase inhibition through pharmacological inhibition or siRNA knockdown. Preventing ganglioside conversion to GM1 inhibited regeneration in PNS axons. However, replacing Neu3 sialidase with external sialidase after blocking it rescued regeneration by inducing an increase in GM1 (and reduction in GD1a). This suggests that PNS axotomy rapidly activates Neu3 sialidase, converting major nervous system gangliosides to GM1. Within $1 \mathrm{~h}$ after axotomy, as axons regenerated their growth cones, GDla and GM1 levels reverted to preaxotomy values, implying that Neu3 sialidase activation is transient. Although regulatory mechanisms

for Neu3 sialidase are unknown, the structure of Neu3 sialidase protein has been characterized, revealing potential phosphorylation sites on the $\mathrm{C}$ terminus (Miyagi et al., 1999). After in vitro axotomy, there is a transient influx of calcium and rapid activation of several signaling pathways, including P38MAPK, ERK, and PI3 kinase (Liu and Snider, 2001; Chierzi et al., 2005; Verma et al., 2005). Our results show that blockade of these pathways prevents Neu3 sialidase activation after axotomy. The first step in Neu3 sialidase activation is calcium influx into the damaged axon which we found to be necessary for GD1a to GM1 ganglioside conversion, and for activation of other signaling pathways. Calcium influx after axotomy initiates several other processes involved in regeneration, including activation of calpain and $\mathrm{Ca}^{2+}$-dependent kinases (Ziv and Spira, 1997; Chierzi et al., 2005; Kamber et al., 2009). The next step we identified was activation of P38MAPK, a stress-related kinase, activated by stressors including calcium (Nozaki et al., 2001). Activation of P38MAPK by anisomycin triggered sialidase activity, confirming P38MAPK as a link between axotomy and Neu3 sialidase activation. However, we do not know whether P38MAPK binds directly to Neu3 sialidase or whether it acts via an intermediary step. Evidence demonstrates that P38MAPK activation is essential for efficient axon regeneration (Myers et al., 2003; Verma et al., 2005; Nix et al., 2011; Kato et al., 2013). Axotomy also activated ERK, which is a step in the major signaling pathway controlling axon growth (Chierzi et al., 2005; Waetzig and Herdegen, 2005). Neu3 sialidase activation and ganglioside conversion is sufficient to activate ERK, confirmed with ERK activation in axons treated with exogenous sialidase. These results suggest that P38MAPK and ERK are acting in parallel within DRG neurons downstream of the initial calcium influx arising immediately after axotomy. This is likely the result of the construction of cell surface signaling domains (rafts), of which GM1 is a major organizer (Ichikawa et al., 2009; Ohkawa et al., 2010; Singh et al., 2010; Furukawa et al., 2011). These domains contain various activators of signaling pathways, such as integrins, growth factor receptors, and other signaling molecules. In embryonic neurons, Neu3 sialidase action can affect axons by increasing TrkA signaling resulting in ERK activation (Da Silva et al., 2005).

\section{Neu3 sialidase activation fails in CNS axons}

Although Neu3 sialidase was activated by axotomy in DRG axons, we observed no such change in adult retinal axons, despite the presence of Neu3 sialidase protein on the axons. These results suggest that there is a failure in the mechanisms that activate Neu3 after axotomy. Where might activation fail? Axotomy of CNS axons causes calcium influx as in PNS axons (Mandolesi et al., 2004), but we did not observe activation of P38MAPK after 
axotomy in retinal axons. Alternatively, if P38MAPK is pharmacologically activated, retinal axons show sialidase activity with ganglioside conversion, suggesting a defect in the pathway leading to P38MAPK activation. Interestingly, axonal regeneration in P38MAPK knock-out mice was shown to be substantially lower than in wild-type mice (Kato et al., 2013). It is not clear why there is a differential regulation of P38MAPK between the two neuronal types; however, we hypothesize that the differential activity profile of RhoA in CNS and PNS axons may partially explain this difference. It has been shown to be activated by the myelin inhibitor Nogo-A (absent in PNS), which acts to suppress P38MAPK levels in CNS neurons after (MCAO) injury (Kilic et al., 2010), inhibiting regeneration. There is also evidence demonstrating that the formation of a complex between GT1b and the Nogo receptor NgR1 inhibits axon growth in cerebellar granular neurons (Williams et al., 2008). Likewise, upregulation of cytokines after injury, such as TNF- $\alpha$, has also been shown to activate RhoA in cultured hippocampal neurons leading to reduced P38MAPK activation (Neumann et al., 2002). However, TNF- $\alpha$ has an opposite effect in DRG neurons leading to increases in after injury calcium influx and activation of P38MAPK (Pollock et al., 2002).

\section{Actions of Neu3 sialidase}

Neu3 sialidase is membrane-associated, and its action is known to be specific to membrane gangliosides (Miyagi et al., 1999). Neu3 sialidase might affect axon regeneration in several ways. In one well-established mechanism, GDla is a coreceptor for the inhibitory molecule MAG, present on PNS- and CNSmyelinating cells (Collins et al., 1997; Vyas et al., 2002). Conversion of GD1a to GM1 therefore diminishes inhibition by MAG. Although important in vivo, where axons and myelinating glia are in close contact, it is not likely to be the mechanism behind the positive effect of sialidase in our in vitro assays as these axons were not in contact with myelinating glia, and inhibition of ganglioside synthesis blocked rather than stimulated axon regeneration. Instead, our results suggest a positive effect of GM1 on axon regeneration. However, in addition to the "growth-promoting effect" of increased GM1 levels, it is possible that the increase in regeneration was also the result of a reduction of complex gangliosides, such as GD1a or GT1b. All interventions that increased axonal GM1 increased regeneration and those that inhibited conversion of polysialylated gangliosides to GM1 blocked regeneration. This conclusion is strengthened by our results with axotomized retinal axons, which do not upregulate GM1 ganglioside and fail to regenerate unless treated with sialidase to convert surface gangliosides to GM1. Potential mechanisms have been proposed to explain how GM1 might enable growth cone regeneration, mostly based on the ability of GM1 to organize lipid-rich microdomains/rafts (Furukawa et al., 2011). Disruption of one type of raft through manipulation of flotillins affects regeneration, for example (Stuermer, 2010). Additionally, extensive literature on cancer invasion and Neu3 sialidase exists showing that Neu3 sialidase is localized to membrane ruffles in GM1-rich rafts during metastasis (Yamaguchi et al., 2006; Miyagi et al., 2008a,b; Miyata et al., 2011; Tringali et al., 2012). Metastatic transformation and growth cone advance share a requirement for membrane extension, decreased adhesiveness to substrate at the leading edge, and formation of fillipodia and lamellipodia. In tumor cells, Neu3 sialidase has effects on adhesion, integrin turnover and activation, caspase activity, caveolins, and several signaling pathways (Roche et al., 1997; Miyagi et al., 2008a,b; Tringali et al., 2012). Disruption of microdomains/rafts might explain why mice lacking complex gangliosides have decreased expression of neurotrophic factors and their receptors (Kittaka et al., 2008), and why GM1 ganglioside potentiates the activity of NGF receptor, TrkA, in addition to other neurotrophin receptors (Bähr et al., 1989; Cuello et al., 1994; Rabin and Mocchetti, 1995; Rabin et al., 2002). Additionally, it is likely that Neu3 sialidase activation may lead to its association with integrins, further leading to activation of FAK and calcium influx (Wu et al., 2007), an event important for cytoskeletal reorganization and axonal guidance (Kato et al., 2006; Miyagi et al., 2008b).

The experiments in this paper demonstrate that an increase in the ratio of growth-promoting gangliosides (GM1) to growthinhibiting gangliosides (such as GD1a and GT1b) mediated by Neu3 sialidase play an important role in changes that lead to axon regeneration after axotomy. Much of the detail about cytoskeletal changes that occur after axotomy is known (Spira et al., 2003; Erez et al., 2007; Ertürk et al., 2007), and we are beginning to understand the process of local mRNA translation (Hanz et al., 2003; Willis et al., 2005; Vogelaar et al., 2009; Yoo et al., 2010; Jung et al., 2012). Equally important are changes on the cell surface, and our results strongly suggest that understanding these events will enable interventions that enhance the intrinsic regenerative ability of axons.

\section{References}

Abad-Rodriguez J, Robotti A (2007) Regulation of axonal development by plasma membrane gangliosides. J Neurochem 103:47-55. CrossRef Medline

Abe N, Borson SH, Gambello MJ, Wang F, Cavalli V (2010) Mammalian target of rapamycin (mTOR) activation increases axonal growth capacity of injured peripheral nerves. J Biol Chem 285:28034-28043. CrossRef Medline

Afshari FT, Kappagantula S, Fawcett JW (2009) Extrinsic and intrinsic factors controlling axonal regeneration after spinal cord injury. Expert Rev Mol Med 11:e37. CrossRef Medline

Agthong S, Koonam J, Kaewsema A, Chentanez V (2009) Inhibition of MAPK ERK impairs axonal regeneration without an effect on neuronal loss after nerve injury. Neurol Res 31:1068-1074. CrossRef Medline

Agthong S, Kaewsema A, Chentanez V (2012) Inhibition of P38 MAPK reduces loss of primary sensory neurons after nerve transection. Neurol Res 34:714-720. CrossRef Medline

Bähr M, Vanselow J, Thanos S (1989) Ability of adult rat ganglion cells to regrow axons in vitro can be influenced by fibroblast growth factor and gangliosides. Neurosci Lett 96:197-201. CrossRef Medline

Bradke F, Dotti CG (1999) The role of local actin instability in axon formation. Science 283:1931-1934. CrossRef Medline

Bradke F, Fawcett JW, Spira ME (2012) Assembly of a new growth cone after axotomy: the precursor to axon regeneration. Nat Rev Neurosci 13:183193. CrossRef Medline

Chew DJ, Fawcett JW, Andrews MR (2012) The challenges of long-distance axon regeneration in the injured CNS. Prog Brain Res 201:253-294. CrossRef Medline

Chierzi S, Ratto GM, Verma P, Fawcett JW (2005) The ability of axons to regenerate their growth cones depends on axonal type and age, and is regulated by calcium, cAMP and ERK. Eur J Neurosci 21:2051-2062. CrossRef Medline

Cho Y, Cavalli V (2012) HDAC5 is a novel injury-regulated tubulin deacetylase controlling axon regeneration. EMBO J 31:3063-3078. CrossRef Medline

Cho Y, Sloutsky R, Naegle KM, Cavalli V (2013) Injury-induced HDAC5 nuclear export is essential for axon regeneration. J Neurosci 155:894-908. CrossRef

Collins BE, Yang LJ, Mukhopadhyay G, Filbin MT, Kiso M, Hasegawa A, Schnaar RL (1997) Sialic acid specificity of myelin-associated glycoprotein binding. J Biol Chem 272:1248-1255. CrossRef Medline

Coulthard LR, White DE, Jones DL, McDermott MF, Burchill SA (2009) P38(MAPK): stress responses from molecular mechanisms to therapeutics. Trends Mol Med 15:369-379. CrossRef Medline

Cuello AC, Garofalo L, Liberini P, Maysinger D (1994) Cooperative effects of gangliosides on trophic factor-induced neuronal cell recovery and syn- 
aptogenesis: studies in rodents and subhuman primates. Prog Brain Res 101:337-355. CrossRef Medline

Da Silva JS, Hasegawa T, Miyagi T, Dotti CG, Abad-Rodriguez J (2005) Asymmetric membrane ganglioside sialidase activity specifies axonal fate. Nat Neurosci 8:606-615. CrossRef Medline

Erez H, Malkinson G, Prager-Khoutorsky M, De Zeeuw CI, Hoogenraad CC, Spira ME (2007) Formation of microtubule-based traps controls the sorting and concentration of vesicles to restricted sites of regenerating neurons after axotomy. J Cell Biol 176:497-507. CrossRef Medline

Ertürk A, Hellal F, Enes J, Bradke F (2007) Disorganized microtubules underlie the formation of retraction bulbs and the failure of axonal regeneration. J Neurosci 27:9169-9180. CrossRef Medline

Facci L, Leon A, Toffano G, Sonnino S, Ghidoni R, Tettamanti G (1984) Promotion of neuritogenesis in mouse neuroblastoma cells by exogenous gangliosides: relationship between the effect and the cell association of ganglioside GM1. J Neurochem 42:299-305. CrossRef Medline

Furukawa K, Ohmi Y, Ohkawa Y, Tokuda N, Kondo Y,Tajima O, Furukawa K (2011) Regulatory mechanisms of nervous systems with glycosphingolipids. Neurochem Res 36:1578-1586. CrossRef Medline

Gao X, Lowry PR, Zhou X, Depry C, Wei Z, Wong GW, Zhang J (2011) PI3K/Akt signaling requires spatial compartmentalization in plasma membrane microdomains. Proc Natl Acad Sci U S A 108:14509-14514. CrossRef Medline

Geisler FH, Coleman WP, Grieco G, Poonian D (2001) The Sygen multicenter acute spinal cord injury study. Spine 26:S87-S98. CrossRef Medline

Grider MH, Park D, Spencer DM, Shine HD (2009) Lipid raft-targeted Akt promotes axonal branching and growth cone expansion via mTOR and Rac1, respectively. J Neurosci Res 87:3033-3042. CrossRef Medline

Guirland C, Zheng JQ (2007) Membrane lipid rafts and their role in axon guidance. Adv Exp Med Biol 621:144-155. CrossRef Medline

Guirland C, Suzuki S, Kojima M, Lu B, Zheng JQ (2004) Lipid rafts mediate chemotropic guidance of nerve growth cones. Neuron 42:51-62. CrossRef Medline

Hanz S, Perlson E, Willis D, Zheng JQ, Massarwa R, Huerta JJ, Koltzenburg M, Kohler M, van-Minnen J, Twiss JL, Fainzilber M (2003) Axoplasmic importins enable retrograde injury signaling in lesioned nerve. Neuron 40:1095-1104. CrossRef Medline

Ichikawa N, Iwabuchi K, Kurihara H, Ishii K, Kobayashi T, Sasaki T, Hattori N, Mizuno Y, Hozumi K, Yamada Y, Arikawa-Hirasawa E (2009) Binding of laminin-1 to monosialoganglioside GM1 in lipid rafts is crucial for neurite outgrowth. J Cell Sci 122:289-299. CrossRef Medline

Jung H, Yoon BC, Holt CE (2012) Axonal mRNA localization and local protein synthesis in nervous system assembly, maintenance and repair. Nat Rev Neurosci 13:308-324. CrossRef Medline

Kalka D, von Reitzenstein C, Kopitz J, Cantz M (2001) The plasma membrane ganglioside sialidase cofractionates with markers of lipid rafts. Biochem Biophys Res Commun 283:989-993. CrossRef Medline

Kamber D, Erez H, Spira ME (2009) Local calcium-dependent mechanisms determine whether a cut axonal end assembles a retarded endbulb or competent growth cone. Exp Neurol 219:112-125. CrossRef Medline

Kamiguchi H (2006) The region-specific activities of lipid rafts during axon growth and guidance. J Neurochem 98:330-335. CrossRef Medline

Kato K, Shiga K, Yamaguchi K, Hata K, Kobayashi T, Miyazaki K, Saijo S, Miyagi T (2006) Plasma-membrane-associated sialidase (Neu3) differentially regulates integrin-mediated cell proliferation through lamininand fibronectin-derived signalling. Biochem J 394:647-656. CrossRef Medline

Kato N, Matsumoto M, Kogawa M, Atkins GJ, Findlay DM, Fujikawa T, Oda H, Ogata M (2013) Critical role of P38 MAPK for regeneration of the sciatic nerve following crush injury in vivo. J Neuroinflammation 10:1. CrossRef Medline

Katoh-Semba R, Kaneko R, Kitajima S, Tsuzuki M, Ichisaka S, Hata Y, Yamada H, Miyazaki N, Takahashi Y, Kato K (2009) Activation of P38 mitogen-activated protein kinase is required for in vivo brain-derived neurotrophic factor production in the rat hippocampus. Neuroscience 163:352-361. CrossRef Medline

Kilic E, ElAli A, Kilic U, Guo Z, Ugur M, Uslu U, Bassetti CL, Schwab ME, Hermann DM (2010) Role of Nogo-A in neuronal survival in the reperfused ischemic brain. J Cereb Blood Flow Metab 30:969-984. CrossRef Medline

Kittaka D, Itoh M, Ohmi Y, Kondo Y, Fukumoto S, Urano T, Tajima O,
Furukawa K (2008) Impaired hypoglossal nerve regeneration in mutant mice lacking complex gangliosides: down-regulation of neurotrophic factors and receptors as possible mechanisms. Glycobiology 18:509-516. CrossRef Medline

Kopitz J, von Reitzenstein C, Sinz K, Cantz M (1996) Selective ganglioside desialylation in the plasma membrane of human neuroblastoma cells. Glycobiology 6:367-376. CrossRef Medline

Lehmann HC, Lopez PH, Zhang G, Ngyuen T, Zhang J, Kieseier BC, Mori S, Sheikh KA (2007) Passive immunization with anti-ganglioside antibodies directly inhibits axon regeneration in an animal model. J Neurosci 27:27-34. CrossRef Medline

Liu RY, Snider WD (2001) Different signaling pathways mediate regenerative versus developmental sensory axon growth. J Neurosci 21:RC164. Medline

Long KE, Lemmon V (2000) Dynamic regulation of cell adhesion molecules during axon outgrowth. J Neurobiol 44:230-245. CrossRef Medline

Lopez PH, Zhang G, Zhang J, Lehmann HC, Griffin JW, Schnaar RL, Sheikh KA (2010) Passive transfer of IgG anti-GM1 antibodies impairs peripheral nerve repair. J Neurosci 30:9533-9541. CrossRef Medline

Mandolesi G, Madeddu F, Bozzi Y, Maffei L, Ratto GM (2004) Acute physiological response of mammalian central neurons to axotomy: ionic regulation and electrical activity. FASEB J 18:1934-1936. CrossRef Medline

Mei S, Gu H, Ward A, Yang X, Guo H, He K, Liu Z, Cao W (2012) P38 mitogen-activated kinase (MAPK) promotes cholesterol ester formation accumulation in macrophages through inhibition of macroautophagy. J Biol Chem 15:11761-11768. CrossRef Medline

Miyagi T, Wada T, Iwamatsu A, Hata K, Yoshikawa Y, Tokuyama S, Sawada M (1999) Molecular cloning and characterization of a plasma membrane-associated sialidase specific for gangliosides. J Biol Chem 274: 5004-5011. CrossRef Medline

Miyagi T, Wada T, Yamaguchi K (2008a) Roles of plasma membraneassociated sialidase Neu3 in human cancers. Biochim Biophys Acta 1780: 532-537. CrossRef Medline

Miyagi T, Wada T, Yamaguchi K, Hata K, Shiozaki K (2008b) Plasma membrane-associated sialidase as a crucial regulator of transmembrane signalling. J Biochem 144:279-285. CrossRef Medline

Miyata M, Kambe M, Tajima O, Moriya S, Sawaki H, Hotta H, Kondo Y, Narimatsu H, Miyagi T, Furukawa K, Furukawa K (2011) Membrane sialidase Neu 3 is highly expressed in human melanoma cells promoting cell growth with minimal changes in the composition of gangliosides. Cancer Sci 102:2139-2149. CrossRef Medline

Monti E, Miyagi T (2012) Structure and function of mammalian sialidases. Top Curr Chem. Advance online publication. Retrieved May 26, 2012. doi: 10.1007/128_2012_328. CrossRef Medline

Moore DL, Goldberg JL (2011) Multiple transcription factor families regulate axon growth and regeneration. Dev Neurobiol 71:1186-1211. CrossRef Medline

Mountney A, Zahner MR, Lorenzini I, Oudega M, Schramm LP, Schnaar RL (2010) Sialidase enhances recovery from spinal cord contusion injury. Proc Natl Acad Sci U S A 107:11561-11566. CrossRef Medline

Mountney A, Zahner MR, Sturgill ER, Riley CJ, Aston JW, Oudega M, Schramm LP, Hurtado A, Schnaar RL (2013) Sialidase, chondroitinase $\mathrm{ABC}$, and combination therapy after spinal cord contusion injury. J Neurotrauma 30:181-190. CrossRef Medline

Myers RR, Sekiguchi Y, Kikuchi S, Scott B, Medicherla S, Protter A, Campana WM (2003) Inhibition of P38 MAP kinase activity enhances axonal regeneration. Exp Neurol 184:606-614. CrossRef Medline

Neumann H, Schweigreiter R, Yamashita T, Rosenkranz K, Wekerle H, Barde YA (2002) Tumor necrosis factor inhibits neurite outgrowth and branching of hippocampal neurons by a $\rho$-dependent mechanism. J Neurosci 22:854-862. Medline

Nix P, Hisamoto N, Matsumoto K, Bastiani M (2011) Axon regeneration requires coordinate activation of P38 and JNK MAPK pathways. Proc Natl Acad Sci U S A 108:10738-10743. CrossRef Medline

Norambuena A, Schwartz MA (2011) Effects of integrin-mediated cell adhesion on plasma membrane lipid raft components and signaling. Mol Biol Cell 22:3456-3464. CrossRef Medline

Nozaki K, Nishimura M, Hashimoto N (2001) Mitogen-activated protein kinases and cerebral ischemia. Mol Neurobiol 23:1-19. CrossRef Medline

Oehler C, Kopitz J, Cantz M (2002) Substrate specificity and inhibitor studies of a membrane-bound ganglioside sialidase isolated from human brain tissue. Biol Chem 383:1735-1742. CrossRef Medline 
Ohkawa Y, Miyazaki S, Hamamura K, Kambe M, Miyata M, Tajima O, Ohmi Y, Yamauchi Y, Furukawa K, Furukawa K (2010) Ganglioside GD3 enhances adhesion signals and augments malignant properties of melanoma cells by recruiting integrins to glycolipid-enriched microdomains. J Biol Chem 285:27213-27223. CrossRef Medline

Okada M, Itoh Mi M, Haraguchi M, Okajima T, Inoue M, Oishi H, Matsuda Y, Iwamoto T, Kawano T, Fukumoto S, Miyazaki H, Furukawa K, Aizawa S, Furukawa K (2002) b-Series ganglioside deficiency exhibits no definite changes in the neurogenesis and the sensitivity to Fas-mediated apoptosis but impairs regeneration of the lesioned hypoglossal nerve. J Biol Chem 277:1633-1636. CrossRef Medline

Pollock J, McFarlane SM, Connell MC, Zehavi U, Vandenabeele P, MacEwan DJ, Scott RH (2002) TNF-alpha receptors simultaneously activate $\mathrm{Ca}^{2+}$ mobilisation and stress kinases in cultured sensory neurones. Neuropharmacology 42:93-106. CrossRef Medline

Rabin SJ, Mocchetti I (1995) GM1 ganglioside activates the high-affinity nerve growth factor receptor trkA. J Neurochem 65:347-354. CrossRef Medline

Rabin SJ, Bachis A, Mocchetti I (2002) Gangliosides activate Trk receptors by inducing the release of neurotrophins. J Biol Chem 277:49466-49472. CrossRef Medline

Roche PH, Figarella-Branger D, Daniel L, Bianco N, Pellet W, Pellissier JF (1997) Expression of cell adhesion molecules in normal nerves, chronic axonal neuropathies and Schwann cell tumors. J Neurol Sci 151:127-133. CrossRef Medline

Rodriguez JA, Piddini E, Hasegawa T, Miyagi T, Dotti CG (2001) Plasma membrane ganglioside sialidase regulates axonal growth and regeneration in hippocampal neurons in culture. J Neurosci 21:8387-8395. Medline

Sheikh KA, Sun J, Liu Y, Kawai H, Crawford TO, Proia RL, Griffin JW, Schnaar RL (1999) Mice lacking complex gangliosides develop Wallerian degeneration and myelination defects. Proc Natl Acad Sci U S A 96:7532-7537. CrossRef Medline

Singh RD, Marks DL, Holicky EL, Wheatley CL, Kaptzan T, Sato SB, Kobayashi T, Ling K, Pagano RE (2010) Gangliosides and betal-integrin are required for caveolae and membrane domains. Traffic 11:348-360. CrossRef Medline

Skaper SD, Katoh-Semba R, Varon S (1985) GM1 ganglioside accelerates neurite outgrowth from primary peripheral and central neurons under selected culture conditions. Brain Res 355:19-26. Medline

Spira ME, Oren R, Dormann A, Gitler D (2003) Critical calpain-dependent ultrastructural alterations underlie the transformation of an axonal segment into a growth cone after axotomy of cultured Aplysia neurons. J Comp Neurol 457:293-312. CrossRef Medline

Stuermer CA (2010) The reggie/flotillin connection to growth. Trends Cell Biol 20:6-13. CrossRef Medline

Sun F, He Z (2010) Neuronal intrinsic barriers for axon regeneration in the adult CNS. Curr Opin Neurobiol 20:510-518. CrossRef Medline

Tringali C, Lupo B, Silvestri I, Papini N, Anastasia L, Tettamanti G, Venerando $B$ (2012) The plasma membrane sialidase Neu3 regulates the malignancy of renal carcinoma cells by controlling $\beta 1$ integrin internalization and recycling. J Biol Chem 287:42835-42845. CrossRef Medline

Verma P, Chierzi S, Codd AM, Campbell DS, Meyer RL, Holt CE, Fawcett JW
(2005) Axonal protein synthesis and degradation are necessary for efficient growth cone regeneration. J Neurosci 25:331-342. CrossRef Medline

Vogelaar CF, Gervasi NM, Gumy LF, Story DJ, Raha-Chowdhury R, Leung KM, Holt CE, Fawcett JW (2009) Axonal mRNAs: characterisation and role in the growth and regeneration of dorsal root ganglion axons and growth cones. Mol Cell Neurosci 42:102-115. CrossRef Medline

Vyas AA, Patel HV, Fromholt SE, Heffer-Lauc M, Vyas KA, Dang J, Schachner M, Schnaar RL (2002) Gangliosides are functional nerve cell ligands for myelin-associated glycoprotein (MAG), an inhibitor of nerve regeneration. Proc Natl Acad Sci U S A 99:8412-8417. CrossRef Medline

Waetzig V, Herdegen T (2005) MEKK1 controls neurite regrowth after experimental injury by balancing ERK1/2 and JNK2 signaling. Mol Cell Neurosci 30:67-78. CrossRef Medline

Wang Z, Jin Y (2011) Genetic dissection of axon regeneration. Curr Opin Neurobiol 21:189-196. CrossRef Medline

Williams G, Wood A, Williams EJ, Gao Y, Mercado ML, Katz A, JosephMcCarthy D, Bates B, Ling HP, Aulabaugh A, Zaccardi J, Xie Y, Pangalos MN, Walsh FS, Doherty P (2008) Ganglioside inhibition of neurite outgrowth requires Nogo receptor function: identification of interaction sites and development of novel antagonists. J Biol Chem 283:16641-16652. CrossRef Medline

Willis D, Li KW, Zheng JQ, Chang JH, Smit AB, Kelly T, Merianda TT, Sylvester J, van Minnen J, Twiss JL (2005) Differential transport and local translation of cytoskeletal, injury-response, and neurodegeneration protein mRNAs in axons. J Neurosci 25:778-791. CrossRef Medline

Witte H, Neukirchen D, Bradke F (2008) Microtubule stabilization specifies initial neuronal polarization. J Cell Biol 180:619-632. CrossRef Medline

Wu G, Lu ZH, Obukhov AG, Nowycky MC, Ledeen RW (2007) Induction of calcium influx through TRPC 5 channels by cross-linking of GM1 ganglioside associated with alpha5betal integrin initiates neurite outgrowth. J Neurosci 27:7447-7458. CrossRef Medline

Yamaguchi K, Hata K, Wada T, Moriya S, Miyagi T (2006) Epidermal growth factor-induced mobilization of a ganglioside-specific sialidase (Neu3) to membrane ruffles. Biochem Biophys Res Commun 346:484490. CrossRef Medline

Yang LJ, Lorenzini I, Vajn K, Mountney A, Schramm LP, Schnaar RL (2006) Sialidase enhances spinal axon outgrowth in vivo. Proc Natl Acad Sci U S A 103:11057-11062. CrossRef Medline

Yoo S, van Niekerk EA, Merianda TT, Twiss JL (2010) Dynamics of axonal mRNA transport and implications for peripheral nerve regeneration. Exp Neurol 223:19-27. CrossRef Medline

Zhang G, Lehmann HC, Manoharan S, Hashmi M, Shim S, Ming GL, Schnaar RL, Lopez PH, Bogdanova N, Sheikh KA (2011) Anti-ganglioside antibody-mediated activation of RhoA induces inhibition of neurite outgrowth. J Neurosci 31:1664-1675. CrossRef Medline

Zheng JQ, Kelly TK, Chang B, Ryazantsev S, Rajasekaran AK, Martin KC, Twiss JL (2001) A functional role for intra-axonal protein synthesis during axonal regeneration from adult sensory neurons. J Neurosci 21:92919303. Medline

Ziv NE, Spira ME (1997) Localized and transient elevations of intracellular $\mathrm{Ca}^{2+}$ induce the dedifferentiation of axonal segments into growth cones. J Neurosci 17:3568-3579. Medline 